\title{
The geometry of causal probability trees that are algebraically constrained
}

\author{
Eva Riccomagno ${ }^{1,2}$ and Jim. Q. Smith ${ }^{2}$ \\ 1 Department of Mathematics, Polytechnic of Turin, Corso Duca degli Abruzzi, \\ 24, 10129, Torino, Italy Eva.Riccomagno@polito.it \\ 2 Department of Statistics, The University of Warwick, Gibbet Hill Road, CV4 \\ 7AL, UK J.Q.Smith@warwick.ac.uk
}

In this chapter we show how algebraic geometry can be used to define and then analyse the properties of certain important classes of discrete probability models described through probability trees. Our aim is to show how much wider classes of discrete statistical models than have been considered previously have a useful algebraic formulation and unlike its competitors this class is closed under discovery of arbitrary marginal distributions. We proceed to illustrate how the identifiability of certain causal functions can be articulated and analysed within this framework which generalises the causal Bayesian network formulation. We note that as with causal Bayesian networks the most convenient polynomial parametrisation is one that is based on conditional rather than marginal probabilities.

In Section 1 we introduce the probability tree representation of a discrete model and show that discrete Bayesian networks and some of their recent generalisations are special subclasses of these models. We then introduce an algebraic representation of important classes of probability tree models called algebraic constraint models (ACTs). In Section 3 we proceed to examine how ACTs are closed under the discovery of the marginal distribution of a random variable measurable with respect to the path sigma algebra of its underlying probability tree. Probability tree representations are especially useful to specify and study the implications of certain causal hypotheses. In Sections 4 and 5 we relate these causal models to ACTs and give a formal discussion of the conditional probability graphs of discrete models that can also be expressed as Bayesian networks. In Section 6 we illustrate these ideas from the perspective of a simple modelling context.

\section{The algebra of probability trees}

Begin by considering a finite discrete probability space whose atomic events are given by the $N$ root to leaf paths on a probability tree $\mathcal{T}=(V(\mathcal{T}), E(\mathcal{T}))$, 
where $V(\mathcal{T})$ and $E(\mathcal{T})$ are the vertex set and edge set, respectively, and $\mathcal{T}$ has a single root vertex $v_{0}$. Then an atomic event is a path $\lambda$ with $n(\lambda)$ edges $\left(e_{1}(\lambda), e_{2}(\lambda), \ldots e_{n(\lambda)}(\lambda)\right)$ and $n(\lambda)+1$ respective vertices $\left(v_{0}, v_{1}(\lambda), \ldots v_{n(\lambda)}(\lambda)\right)$. In the listing of the edges and vertices of a path we might suppress the index $\lambda$ when there is no ambiguity. To each path we associate the probability

$$
p(\lambda)=\prod_{i=1}^{n(\lambda)} \pi_{i}\left(e_{i}(\lambda)\right)
$$

where $\pi\left(e_{i}(\lambda)\right)$ is a probability on the edge $e_{i}(\lambda)$ for $1 \leq i \leq n(\lambda)$.

A non leaf vertex is called a situation and $S(\mathcal{T}) \subset V(\mathcal{T})$ is the set of situations. To each situation $v \in S(\mathcal{T})$ we associate a random variable $X(v)$ whose sample space, $\mathbb{X}(v)$, can be labeled by the edges emanating from $v$, equivalently by the children of $v$ in $\mathcal{T}$. Let $E(v) \subseteq E(\mathcal{T})$ be the set of edges out of $v$. Here without loss of generality we assume that the random variables in $\{X(v): v \in S(\mathcal{T})\}$ are mutually independent of each other. Clearly $0 \leq$ $\pi(e) \leq 1$ for all $e \in E(v)$ and all $v \in S(\mathcal{T})$. Moreover for all $v \in S(\mathcal{T})$

$$
\sum_{e \in E(v)} \pi(e)=1
$$

i.e. the set of probabilities on edges emanating from each situation in the tree satisfy simplex conditions. Call the set of probabilities

$$
\Pi=\{\pi(e): e \in E(v) \text { and } v \in S(\mathcal{T})\}
$$

the set of primitive probabilities of $\mathcal{T}$. An equivalent notation for primitive probabilities highlights the vertices of the edge [21], namely for the edge $e=$ $\left(v, v^{\prime}\right)$ where $v$ is the out vertex of $e$ and $v^{\prime}$ is its in vertex,

$$
\left\{\pi(e)=\pi\left(v^{\prime} \mid v\right)=\operatorname{Prob}\left(X(v)=v^{\prime}\right): v^{\prime} \in \mathbb{X}(v) \text { and } v \in S(\mathcal{T})\right\}
$$

A simple probability tree is given in Table 1 . The set of situation is $S(\mathcal{T})=$ $\left\{v_{i}: 0 \leq i \leq 4\right\}$ and the random variable $X\left(v_{i}\right)$ is associated to the situation $v_{i} \in S(\mathcal{T})$, for $0 \leq i \leq 5$. The random variables $X\left(v_{i}\right), 0 \leq i \leq 5$, have state spaces of dimensions $3,2,3,2,2$ respectively. We can label each edge $e_{i} \in E(\mathcal{T})$ by its receiving vertex. So for example we label the edge $\left(v_{2}, v_{8}\right)$ as $e_{8}$. Each edge $e_{i} \in E(\mathcal{T})$ has an associated primitive probability $\pi\left(e_{i}\right)$ which we will write as $\pi_{i}, 1 \leq i \leq 12$. The eight root to leaf paths $\lambda$ of $\mathcal{T}$ can be indexed by the index of their terminal vertex. So for example $\lambda_{12}=\left(v_{0}, v_{1}, v_{4}, v_{12}\right)$. The probabilities $p_{i}=\operatorname{Prob}\left(\lambda_{i}\right)$ of these atomic events $\lambda_{i}, 5 \leq i \leq 12$ are then given by the following monomials in the primitive probabilities

$$
\begin{aligned}
& p_{5}=\pi_{1} \pi_{5}, p_{6}=\pi_{2} \pi_{6}, \quad p_{7}=\pi_{2} \pi_{7} \quad, p_{8}=\pi_{2} \pi_{8} \\
& p_{9}=\pi_{3} \pi_{9}, p_{10}=\pi_{3} \pi_{10}, p_{11}=\pi_{1} \pi_{4} \pi_{11}, p_{12}=\pi_{1} \pi_{4} \pi_{12}
\end{aligned}
$$

under the linear constraints 


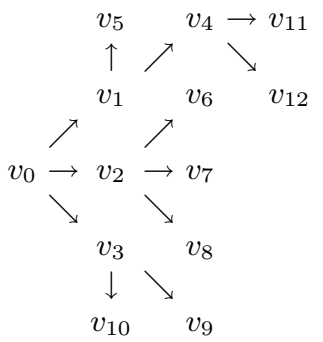

Table 1. A simple probability tree

$$
\begin{aligned}
\pi_{1}+\pi_{2}+\pi_{3} & =1 \\
\pi_{4}+\pi_{5} & =1 \\
\pi_{6}+\pi_{7}+\pi_{8} & =1 \\
\pi_{9}+\pi_{10} & =1 \\
\pi_{11}+\pi_{12} & =1
\end{aligned}
$$

and the inequalities $0 \leq \pi_{i} \leq 1, i=1, \ldots, 12$.

It follows that if we ignore the inequality statements, a discrete probability model represented by a probability tree can be thought of as $N$ monomials in primitive probabilities that satisfy a collection of sum to one conditions where, again, $N$ is the number of atomic events and root to leaf paths. In particular to each saturated probability tree model can be associated a set of polynomial functions satisfying certain linear equations.

Of course, a probability tree model is not usually saturated. Typically certain other constraints are placed on the primitive probabilities of the probability space. These constraints define a particular statistical model. However it is very common that such constraints continue to be algebraic in the primitive probabilities and so form an algebraic variety.

Example 1 (Chain Event Graph Models). These models simply equate certain primitive probabilities. Specifically we say that two situations $v_{1}$ and $v_{2}$ in a probability tree $T$ are in the same stage $u$ if there is an invertible map $\Phi: E\left(v_{1}\right) \rightarrow E\left(v_{2}\right)$ such that, for all $e\left(v_{1}\right) \in E\left(v_{1}\right)$

$$
\pi\left(\Phi\left(e\left(v_{1}\right)\right)=\pi\left(e\left(v_{1}\right)\right)\right.
$$

Note that an equivalent condition would state that $v_{1}, v_{2} \in u$ if and only if there is an invertible function $\Psi$ such that the distribution of $X\left(v_{2}\right)$ is the distribution of $\Psi\left(X\left(v_{1}\right)\right)$. Such models where the probability edges emanating from a given situation are assumed to have the same probabilities are extremely common: see below. Note that algebraically such models simply supplement the linear conditions associated with the saturated tree with 
the linear relationships where we simply identify certain primitive probabilities with each other. For example in the tree in Table 1 we might have that all its situations lay in one of two stages $\left\{u_{1}, u_{2}\right\}$ where $u_{1}=\left\{v_{0}, v_{2}\right\}$ and $u_{2}=\left\{v_{1}, v_{3}, v_{4}\right\}$ and specifically

$$
\begin{aligned}
& \pi_{1}=\pi_{6}, \quad \pi_{2}=\pi_{7}, \quad \pi_{3}=\pi_{8} \\
& \pi_{4}=\pi_{9}=\pi_{11}, \pi_{5}=\pi_{10}=\pi_{12}
\end{aligned}
$$

Substituting, our polynomials become

$$
\begin{aligned}
& p_{5}=\pi_{1} \pi_{5} \quad p_{6}=\pi_{1} \pi_{2} \quad p_{7}=\pi_{2}^{2} \quad p_{8}=\pi_{2} \pi_{3} \\
& p_{9}=\pi_{3} \pi_{4} p_{10}=\pi_{3} \pi_{5} p_{11}=\pi_{1} \pi_{4}^{2} \\
& p_{12}=\pi_{1} \pi_{4} \pi_{5}
\end{aligned}
$$

where

$$
\begin{array}{r}
\pi_{1}+\pi_{2}+\pi_{3}=1 \\
\pi_{4}+\pi_{5}=1
\end{array}
$$

Example 2 (Bayesian Networks). Recently the geometry associated with the algebraic features of finite discrete Bayesian Networks (BNs) have been vigorously studied. See for example $[20,8,9]$. This class of models is a special subclass of Chain Event Graph Models. These have associated probability trees whose root to leaf paths are all the same length, whose valency of vertices the same distance from the root are all identical, and whose stages are only allowed to contain situations the same distance from the root vertex of the tree. The probability tree of the simplest, non degenerate, Bayesian network $X_{1} \rightarrow X_{2} \rightarrow X_{3}$, where $X_{1}, X_{2}, X_{3}$ are all binary is given in Table 2 : In

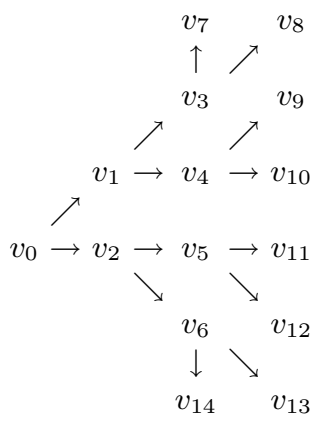

Table 2. Probability tree for the Bayesian network $X_{1} \rightarrow X_{2} \rightarrow X_{3}$

particular $X\left(v_{0}\right)=X_{1}$ and $X\left(v_{1}\right)=\left\{X_{2} \mid X_{1}=0\right\}$ and the full identification is deduced by the following. As the atoms of the sample space are $\left(x_{1}, x_{2}, x_{3}\right)$ where $x_{i}=0,1$ for $1 \leq i \leq 3$, then the eight atomic paths are identified by 


$$
\begin{aligned}
& v_{7}=(0,0,0), \quad v_{8}=(0,0,1), \quad v_{9}=(0,1,0), \quad v_{10}=(0,1,1) \\
& v_{11}=(1,0,0), v_{12}=(1,0,1), v_{13}=(1,1,0), v_{714}=(1,1,1)
\end{aligned}
$$

using the indexing outlined above. Note that for example the edge $e_{5}=\left(v_{2}, v_{5}\right)$ can be associated with the conditional event $\left\{X_{2}=0 \mid X_{1}=1\right\}$. Since the Bayesian network is equivalent to specifying $\left\{X_{3} \sqcup X_{1} \mid X_{2}\right\}$ this model is equivalent to the saturated model given by the tree above together with the four linear constraints

$$
\operatorname{Prob}\left(X_{3}=x_{3} \mid X_{1}=0, X_{2}=x_{2}\right)=\operatorname{Prob}\left(X_{3}=x_{3} \mid X_{1}=1, X_{2}=x_{2}\right)
$$

for $x_{2}, x_{3}=0,1$ which in terms of primitive probabilities reduce to

$$
\pi_{7}=\pi_{11}, \pi_{8}=\pi_{12}, \pi_{9}=\pi_{13}, \pi_{10}=\pi_{14}
$$

Example 3 (Generalisations of Bayesian Networks). As the use of Bayesian networks increased it became increasingly apparent that there were many circumstances when more symmetries could be demanded of a model than could be conveniently expressed as sets of conditional independence models. The stages of the corresponding chain event graphs where subsequently not only being determined by specific parent configurations but also unions of such classes: see for example [[28] ref]. Furthermore the demand for the sample spaces associated with different configurations of parents to have the same dimension given their distance from the root vertex was also seen to be overly restrictive. Thus zeros in the primitives have been systematically modelled and structures built to efficiently process such information in these heterogeneous structures. Formally here we are simply adding further linear constraints that for $\left\{\pi(e)=0: e \in E^{*}(\mathcal{T}) \subset E(\mathcal{T})\right\}$ for some subset $E^{*}(\mathcal{T})$ of edges. Note that from an algebraic point of view all these models are special cases of chain event graph models all with equal length root to leaf paths.

Example 4 (Bayesian Linear Constraint Models). This class of models, first discussed in a rather restricted form in [23], imposes on the saturated tree additional general linear constraints on the primitive probabilities. These arise quite naturally for example, from constrained chain event graph models or such models uncertainly observed but with a known sampling distribution: see below.

Example 5 (Algebraic Parametric Models). These models assume that the probability distributions indexed by the stages of a probability tree lie in a parametric family each of whose primitive probabilities can be expressed as an algebraic function of the hyperparameters of the family. These naturally arise in certain genetic models and also apply when there is good reason to assume that distributions associated with stages come from certain parametric families, the elements of whose probability mass function can be expressed algebraically. Examples of such families of distributions are the Binomial and censored Negative Binomial. In the chain event graph model of Example 1 we 
might be able to assume that $X\left(v_{0}\right)$ has a Binomial $B i\left(2, \pi_{0}\right)$ distribution so that

$$
\pi_{1}=\left(1-\pi_{0}\right)^{2}, \pi_{2}=2 \pi_{0}\left(1-\pi_{0}\right), \pi_{3}=\pi_{0}^{2}
$$

which gives

$$
\begin{array}{lll}
p_{5}=\left(1-\pi_{0}\right)^{2}\left(1-\pi_{4}\right), & p_{6}=2 \pi_{0}\left(1-\pi_{0}\right)^{3}, & p_{7}=4 \pi_{0}^{2}\left(1-\pi_{0}\right)^{2} \\
p_{8}=2 \pi_{0}^{3}\left(1-\pi_{0}\right), & p_{9}=\pi_{0}^{2} \pi_{4}, & p_{10}=\pi_{0}^{2}\left(1-\pi_{4}\right) \\
p_{11}=\left(1-\pi_{0}\right)^{2} \pi_{4}^{2} & p_{12}=\left(1-\pi_{0}\right)^{2} \pi_{4}\left(1-\pi_{4}\right)
\end{array}
$$

on substituting $\pi_{5}=1-\pi_{4}$.

All these families of discrete models have their atomic probabilities expressed as monomials under linear constraints or as polynomials and motivate the following definition which contains them all.

Definition 1. An algebraically constrained tree (ACT) is a probability tree together with a (possibly null) set of algebraic constraints over its associated primitive probabilities.

We note that standard classes of model like the chain graph models in [30], [11], [13] or more unconventional ones, see e.g. [17] can all be written as ACTs. Also parsimonious multivariate families fall into this class, see for example [7] and [3]. So the classes of models amenable to tree based factorisations are very wide.

An important property of the class of ACTs is that, unlike Bayesian networks and Chain Event Graph Models, they are closed to the imposition of marginal constraints in a sense developed in the next section.

\section{Manifest probabilities and solution spaces}

\subsection{Introduction}

In a discrete statistical model it is common to have information about the marginal distributions of certain random variables. For example in a Bayesian network the marginal distribution of certain subsets of variables can be assumed to be known. Thus let $\Lambda(\mathcal{T})$ be the set of root to leaf path in a probability tree $\mathcal{T}$ and let $N$ be its cardinality. Suppose that the probability distribution of a function $M$ of the original sample space $\Lambda(\mathcal{T})$ is known. Then we know the values of the probabilities on a partition $\digamma=\left\{\Lambda_{1}, \Lambda_{2}, \ldots \Lambda_{N^{*}}\right\}$ of $\Lambda(\mathcal{T})$, with $N^{*} \leq N$. For $1 \leq i \leq N^{*}$, each $\Lambda_{i}$ is a non-empty subset of $\Lambda$ and

$$
p\left(\Lambda_{i}\right)=\sum_{\lambda \in \Lambda_{i}} p(\lambda)
$$

The vector $\boldsymbol{p}=\left[p\left(\Lambda_{1}\right), p\left(\Lambda_{2}\right), \ldots, p\left(\Lambda_{N^{*}}\right)\right]$ is often called the manifest probability vector. It is easier to display row vectors and we omit the transpose vector symbol. 
Note that it is an unfortunate practicality, particularly when data is collected from sample surveys, that data associated with some cells in probability tables will be missing or given as crude aggregates, see e.g. [7]. However we can still discover the distribution of a random variable on an albeit possibly partition which are not derived from cross product structures. It follows that the analysis below, unlike the analogous one based on Bayesian network's extends seamlessly into this scenario.

Suppose we are interested in making inferences about a vector function $\xi(\Pi)$ where $\Pi=\{\pi(e): e \in E(\mathcal{T})\}$ is the set of primitive probabilities. For example, $\xi(\Pi)$ could be the vector of all primitives, some function of particular interest - like another margin - , or an expected utility. Interesting questions to answer are now:

Feasibility Are there any models in our class which are consistent with the known probabilities $\boldsymbol{p}$ ?

Inverse image If there are feasible models, what combinations of values of the primitives are consistent with this information?

Identifiability Is there a unique solution $\widehat{\boldsymbol{\xi}}(\boldsymbol{p})$ of the given function of interest $\xi(\Pi)$ in terms of primitives consistent with the discovered probabilities on the partition $\digamma$ ?

Various authors have attempted to answer these questions for Bayesian networks using graphical criteria, [8] and [26], undirected graphical models [9, $28]$ and other algebraic models e.g. [14]. It can checked that all these questions can be framed as queries about the existence and form of a solution space of simultaneous polynomial equations in primitive probabilities in subclasses of ACTs.

It is important however to note that there are two features that make this problem slightly non-standard in algebraic geometry. First the field over which the question is answered is the set of real number rather than complex. Second, to allow that probabilities must be non-negative, we need to intersect the algebraic solutions with the convex space whose hull is determined by the linear inequalities $\pi(e) \geq 0$ for all $e \in E(\mathcal{T})$.

Example 6 (Cont. of Examples 2 and 5). Suppose we simply learn the probability $p_{8}$ of the indicator on the atom $\lambda_{8}$ in the example of an algebraic parametric model given in Example 5. Then since

$$
p_{8}=2 \pi_{0}^{3}\left(1-\pi_{0}\right) \leq 128^{-1} .27 \approx 0.21
$$

the feasible region for $\boldsymbol{p}$ is simply that $0 \leq p_{8} \leq 0.21$. If $p_{8}$ lies outside the feasible region the solution space is clearly empty. If $p_{8}$ lies in the feasible region then when $\xi(\Pi)=\left[\pi_{0}, \pi_{4}\right]$ the solution space is given by $\left\{\left(\pi_{0}, \pi_{4}\right)\right.$ : $\pi_{0}=\widehat{\alpha}_{1}$ or $\left.\widehat{\alpha}_{2}, 0 \leq \pi_{4} \leq 1\right\}$ where $\widehat{\alpha}_{1}, \widehat{\alpha}_{2}$ are the two solutions of the equation

$$
\pi_{0}^{4}-\pi_{0}^{3}+0.5 p_{8}=0
$$


The model is therefore unidentified. Now suppose we learn the distribution of a variable taking different values on $\left\{\left\{\lambda_{8}\right\},\left\{\lambda_{9}\right\}, \Lambda \backslash\left\{\left\{\lambda_{8}, \lambda_{9}\right\}\right\}\right.$ i.e. we learn the values of $p_{8}$ and $p_{9}$. Then it is straightforward to check that the feasible region is now $\left\{\boldsymbol{p}: 0 \leq p_{9} \leq 4 p_{8}, 0 \leq p_{8} \leq 0.21\right\}$. The solution space is then

$$
\left\{\left(\pi_{0}, \pi_{4}\right): \pi_{4}=2 p_{9} p_{8}^{-1} \pi_{0}\left(1-\pi_{0}\right) \text { where } \pi_{0}=\widehat{\alpha}_{1} \text { or } \widehat{\alpha}_{2}\right\}
$$

This is identifiable only when $\widehat{\alpha}_{1}=\widehat{\alpha}_{2}$, that is only when $p_{8}=128^{-1} .27$ and $\pi_{0}=3 / 4$. Note that the complicated nature of the feasible regions is due both to the fact that we are solving over the real numbers and to the fact that primitive probabilities are constrained to be positive.

The nature of solutions to the simple example above is typical and reflected in more common classes of models, see for example [26, 16]. However when models have more arguments it becomes necessary to use sophisticated elimination techniques or to derive characterisation theorems.

\subsection{The closure of ACTs to filtered observations}

It is well known that a Bayesian network $\mathcal{G}$ on a set of random variables $\left\{X_{1}, X_{2}, \ldots, X_{n}\right\}$ is not closed under arbitrary sampling. For example discovering the probability distribution of the linear random variable $Y=\sum_{i=1}^{n} a_{i} X_{i}$ where $a_{i}>0,1 \leq i \leq n$, will typically destroy all the conditional independence expressed in the topology of $\mathcal{G}$ when this distribution is not known. The only functions which can in general preserve conditional independence factorisations are functions of subsets of the original variables. Similarly the class of chain event graphs is only closed to discovering an (albeit much larger) subclass of marginal distributions of certain variables measurable with respect to the underlying tree. On the other hand it is immediate that the class of ACTs are closed under the discovery of the distribution of arbitrary measurable functions $Y$ on the underlying tree. Let $\mathbb{Y}$ be the sample space of $Y$ and $\Lambda_{Y}$ with subsets $\left\{\Lambda_{y}: y \in \mathbb{Y}\right\}$ the partition induced by $Y$ on the root to leaf path set of $\mathcal{T}$. Then discovering the distribution of $Y$ means simply to know the values $q_{Y}(y)$ for each $y \in \mathbb{Y}$, where

$$
q_{Y}(y)=P(Y=y)=\sum_{\lambda \in \Lambda_{y}} p(\lambda)=\sum_{\lambda \in \Lambda_{y}} \prod_{i=1}^{n(\lambda)} \pi\left(e_{i}(\lambda)\right)
$$

For the known vector of real numbers $\boldsymbol{q}_{Y}=[q(y): y \in \mathbb{Y}]$ these can be written as the polynomial equations in primitive probabilities

$$
\left(\sum_{\lambda \in \Lambda_{y}} \prod_{i=1}^{n(\lambda)} \pi_{i}\left(e_{i}(\lambda)\right)\right)-q_{Y}(y)=0
$$


These equations can be simply added to the polynomial constraints specifying the original ACT before the discovery of this margin to give a submodel of the original ACT.

In fact the ACTs are closed to an even wider variety of discovery. Suppose we discover the distribution of a random variable $Z$ taking values $z \in \mathbb{Z}$ and this is a filtered version of $Y$, i.e. the vector of probabilities $\boldsymbol{q}_{Z}=[q(z): z \in \mathbb{Z}]$ is related to the vector of probabilities $\boldsymbol{q}_{Y}=[q(y): y \in \mathbb{Y}]$ through the equation

$$
\boldsymbol{q}_{Z}^{\prime}=M \boldsymbol{q}_{Y}^{\prime}
$$

where $M$ is a known stochastic matrix and $\boldsymbol{q}^{\prime}$ is the transposed vector of $\boldsymbol{q}$. It is easily checked that this again reduces to a set of supplementary polynomial equation embellishing the original family of probability models.

Finally it is sometimes possible through a designed experiment, to discover the values of $\operatorname{Prob}\left(Y_{1}=y_{1} \mid Y_{2}=y_{2}\right)=q\left(y_{1} \mid y_{2}\right)$ for $\left(y_{1}, y_{2}\right) \in A \subseteq \mathbb{Y}_{1} \times \mathbb{Y}_{2}$ and $\operatorname{Prob}\left(Y_{2}=y_{2}\right)>0$. It is easily seen that the value of each of these conditional probabilities is the solution of an algebraic equation which can again be reduced to a polynomial constraint.

This and other closure property make ACT an ideal class of probability models to study algebraically. Of course as we have seen above the more polynomial constraints are added, the higher the chance that the class of models is not feasible i.e. empty. However this is also the case when we extensively elaborate any model.

\subsection{An inferential aside}

As before let $\digamma=\left\{\Lambda_{1}, \Lambda_{2}, \ldots \Lambda_{N^{*}}\right\}$ be the partition of $\Lambda(\mathcal{T})$ induced by the manifest variables. In practice usually the best we can hope to have available is a random sample $\boldsymbol{r}=\left(r_{1}, r_{2}, \ldots r_{N^{*}}\right), \sum_{i=1}^{N^{*}} r_{i}=n$ of units from a multinomial distribution $M n(n, \boldsymbol{p})$ whose likelihood is

$$
l(\boldsymbol{p} \mid \boldsymbol{r})=\prod_{i=1}^{N^{*}} p\left(\Lambda_{i}\right)^{r_{i}}
$$

where $\sum_{i=1}^{N^{*}} p\left(\Lambda_{i}\right)=1$ and $p\left(\Lambda_{i}\right) \geq 0,1 \leq i \leq N^{*}$. Even when the sample size $n$ is enormous and none of the sample counts is small then plugging in the sample proportions $\widehat{\boldsymbol{p}}$ for $\boldsymbol{p}$ is still hazardous. There are issues associated with Borel's Paradox. For example a Bayesian approach to learning the tree probabilities under constraints [25] shows that even in simple problems where parameters are identified the quantity $\widehat{\boldsymbol{\xi}}(\widehat{\boldsymbol{p}})$ may not be uniformly consistent for $\widehat{\boldsymbol{\xi}}(\boldsymbol{p})$. Moreover when samples are of a moderate size the inequality constraints and the algebraic constraints are seriously confounded and the inference, whether Bayesian or classical, is necessarily more complicated. This issue is worthy of a lot more attention than it has so far received. However for simplicity in this paper we will ignore this delicate problem, like Pearl in [19] 
does. Although not telling the whole story, learning the geometry of conditioned spaces, when we know $\boldsymbol{p}$ precisely, certainly gives bounds on how much we can expect to learn about the primitive probabilities and their associated quantities of interest, as well as the likely multiple solutions that might arise.

Next we will address issues associated with being able to identify a particular class of functions $\widehat{\boldsymbol{\xi}}(\boldsymbol{p})$ for a given partition of $\Lambda(\mathcal{T})$, namely those associated with "causal" effects. So first we need to define precisely what we mean by this term.

\section{Expressing causal effects through algebra}

\subsection{Causality defined on probability trees}

There are several ways of parametrising discrete models and some of the simplest to study are those discrete models that can be expressed as undirected graphs. However, as Shafer argues in his book "The Art of Causal Conjecture" [27], the directed parametrisation of probability tables using probability trees is particularly expressive of inferential issues associated with causation: something we will focus on throughout the rest of this work.

The type of causal inference we have in mind can be illustrated as follows. Suppose we have a large random sample from a population whose evolution to an end point of, say, a disease is represented by one of the eight paths of the probability tree $\mathcal{T}=(V(\mathcal{T}), E(\mathcal{T}))$ in Table 1 . We now contemplate imposing a treatment regime on a future sample from the same population. The treatment will take the following form. If a unit takes a path that arrives at a situation $v^{*}$ in a set $V^{*} \subset V(\mathcal{T})$ then that individual will be forced to pass along just one edge $e^{*}=\left(v^{*}, v^{* *}\right) \in E(\mathcal{T})$ and $v^{* *} \in V^{* *}$ for some $V^{* *} \subset V(\mathcal{T})$. This induces new probability values on some edges of the tree. We are often then interested to learn the effect on the disease states were this treatment regime to be imposed on this population. If we could find a formula in terms of the primitive probabilities for the probabilities $\left\{p^{*}(\lambda): \lambda \in \Lambda\right\}$ after this manipulation then we can learn about the effect of the treatment on the population. But what should these $p^{*}$ functions be?

Suppose the probability tree of an ACT has a causal interpretation, so that a situation that happen before another situation always appears closer to the root of the tree. Then there is a very natural set of functions which relates $\left\{p^{*}(\lambda): \lambda \in \Lambda\right\}$ to the set $\Pi$ of primitive probabilities. We can expect the treatment to have no influence on the processes that gave rise to the situations that happen before treatment. Furthermore it may well be reasonable to assume that the subsequent effect (i.e. the effect after $v^{* *}$ ) of intervening and forcing a person who arrives at $v^{*}$ to take the edge $\left(v^{*}, v^{* *}\right)$ will be the same as the effect subsequent to $v^{* *}$ when no intervention takes place. This latter assumption is substantive but often plausible. It demands that the manipulation is local in the sense that the only way the manipulation affects the 
system is where it is enacted and it has no further effect on later probability laws.

Thus in the probability tree in Table 1 consider the manipulation that whenever a unit reaches $v_{1}$, it moves to $v_{4}$ whilst if the unit arrives at $v_{2}$ the unit is treated so that it arrives at $v_{6}$ with certainty. Then we have that $V^{*}=\left\{v_{1}, v_{2}\right\}, V^{* *}=\left\{v_{4}, v_{6}\right\}$. The probabilities on the edges

$$
\left[e_{1}, e_{2}, e_{3}, e_{4}, e_{5}, e_{6}, e_{7}, e_{8}, e_{9}, e_{10}, e_{11}, e_{12}\right]
$$

of the tree under the stage assumptions made in Example 1 are transformed under this manipulation from

$$
\begin{aligned}
& {\left[\pi_{1}, \pi_{2}, \pi_{3}, \pi_{4}, \pi_{5}, \pi_{6}, \pi_{7}, \pi_{8}, \pi_{9}, \pi_{10}, \pi_{11}, \pi_{12}\right]} \\
& =\left[\pi_{1}, \pi_{2}, \pi_{3}, \pi_{4}, \pi_{5}, \pi_{1}, \pi_{2}, \pi_{3}, \pi_{4}, \pi_{5}, \pi_{4}, \pi_{5}\right]
\end{aligned}
$$

to

$$
\begin{aligned}
\pi^{*} & =\left[\pi_{1}, \pi_{2}, \pi_{3}, 1,0,1,0,0, \pi_{9}, \pi_{10}, \pi_{11}, \pi_{12}\right] \\
& =\left[\pi_{1}, \pi_{2}, \pi_{3}, 1,0,1,0,0, \pi_{4}, \pi_{5}, \pi_{4}, \pi_{5}\right]
\end{aligned}
$$

The new manipulated probabilities on atomic events are therefore

$$
\boldsymbol{p}^{*}=\left[0, \pi_{2}, 0,0, \pi_{3} \pi_{9}, \pi_{3} \pi_{10}, \pi_{1} \pi_{11}, \pi_{1} \pi_{12}\right]
$$

If we believe the assumptions in the algebraic model of Example 5, namely $X\left(v_{0}\right)$ has a Binomial $B i\left(2, \pi_{0}\right)$ distribution, and that these are all preserved after the manipulation, then substituting allows this to simplify further to

$$
\boldsymbol{p}^{*}=\left[0,2 \pi_{0}\left(1-\pi_{0}\right), 0,0, \pi_{0}^{2} \pi_{4}, \pi_{0}^{2}\left(1-\pi_{4}\right),\left(1-\pi_{0}\right)^{2} \pi_{4},\left(1-\pi_{0}\right)^{2}\left(1-\pi_{4}\right)\right]
$$

It is important to note that the manipulated probability vector $\boldsymbol{p}^{*}$ is not the same as the vector $\boldsymbol{p}^{\prime}$ of probabilities conditional of the event composed by the set of atoms $\Lambda^{\prime}=\left\{\lambda_{6}, \lambda_{9}, \lambda_{10}, \lambda_{11}, \lambda_{12}\right\}$ consistent with this manipulation. Indeed under the model of Example 5 we note that $\operatorname{Prob}\left(\Lambda^{\prime}\right)=$ $\left(1-\pi_{0}\right)^{2}\left(2 \pi_{0}\left(1-\pi_{0}\right)+\pi_{4}\right)+\pi_{0}^{2}$ so that $\boldsymbol{p}^{\prime}=\left(p_{5}, p_{6}, \ldots, p_{12}\right)$ is given by

$$
\boldsymbol{p}^{\prime}=\frac{\left[0,2 \pi_{0}\left(1-\pi_{0}\right)^{3}, 0,0, \pi_{0}^{2} \pi_{4}, \pi_{0}^{2} \pi_{4}^{2}\left(1-\pi_{4}\right),\left(1-\pi_{0}\right)^{2},\left(1-\pi_{0}\right)^{2} \pi_{4}\left(1-\pi_{4}\right)\right]}{\operatorname{Prob}\left(\Lambda^{\prime}\right)}
$$

In general the relationship between causal probabilities like $\boldsymbol{p}^{*}$ and conditional probabilities like $\boldsymbol{p}^{\prime}$ is a complicated one and in a saturated model can be identified if and only if $\boldsymbol{V}^{*}$ is vertex set of a subtree of $\mathcal{T}$ rooted at $v_{0}$.

Return to the original problem. Suppose the feature of interest is the probability $\xi_{1}(\Pi)$ of the event $\Lambda_{I}=\left\{\lambda_{6}, \lambda_{9}, \lambda_{11}\right\}$ - say, the event associated with the full recovery of the patient - after treatment. This probability is

$$
\begin{aligned}
p_{6}^{*}+p_{9}^{*}+p_{11}^{*} & =2 \pi_{0}\left(1-\pi_{0}\right)+\pi_{0}^{2} \pi_{4}+\left(1-\pi_{0}\right)^{2} \pi_{4} \\
& =\pi_{4}+2\left(1-\pi_{4}\right) \pi_{0}\left(1-\pi_{0}\right)
\end{aligned}
$$

whilst with no treatment it is 


$$
p_{6}+p_{9}+p_{11}=2 \pi_{0}\left(1-\pi_{0}\right)^{3}+\pi_{0}^{2} \pi_{4}+\left(1-\pi_{0}\right)^{2} \pi_{4}^{2}
$$

Now suppose, as above we learn for example $\left(p_{8}, p_{9}\right)$ then substituting we see that whenever $\left(p_{8}, p_{9}\right)$ is feasible

$$
\xi_{1}(\Pi)=p_{6}^{*}+p_{9}^{*}+p_{11}^{*}=\pi_{4}^{0}\left(1+\left(1-\pi_{4}^{0}\right) p_{8} p_{9}^{-1}\right)
$$

where $\pi_{4}^{0}=2 p_{9} p_{8}^{-1} \pi_{0}\left(1-\pi_{0}\right)$ and where $\pi_{0}$ takes the values $\widehat{\alpha}_{1}$ or $\widehat{\alpha}_{2}$. So although $\xi_{1}(\Pi)$ is not identified we know it can take only one of two possible values. So in particular this effect can be bounded above and below. Furthermore under a Bayesian analysis it will have an explicit posterior expectation depending on the weights on $\widehat{\alpha}_{1}$ and $\widehat{\alpha}_{2}$ in the prior density of $\pi_{0}$.

Alternatively we might well be interested in the change in the probability of full recovery $\xi_{2}(\Pi)$ using the treatment rather than doing nothing. We have

$$
\begin{aligned}
\xi_{2}(\Pi) & =p_{6}^{*}+p_{9}^{*}+p_{11}^{*}-p_{6}-p_{9}-p_{11} \\
& =2 \pi_{0}\left(1-\pi_{0}\right)\left(1-\left(1-\pi_{0}\right)^{2}\right)+\left(1-\pi_{0}\right)^{2}\left(1-\pi_{4}^{2}\right) \\
& =\left(1-\pi_{0}\right)\left\{2 \pi_{0}^{2}\left(2-\pi_{0}\right)+\left(1-\pi_{0}\right)\left(1-\pi_{4}^{2}\right)\right\}
\end{aligned}
$$

Again we see that after learning $\left(p_{8}, p_{9}\right)$ we still cannot identify $\xi(\Pi)$, but know it can take only one of two possible values.

$$
\left\{\left(\pi_{0}, \pi_{4}\right): \pi_{4}=2 p_{9} p_{8}^{-1} \pi_{0}\left(1-\pi_{0}\right) \text { where } \pi_{0}=\widehat{\alpha}_{1} \text { or } \widehat{\alpha}_{2}\right\}
$$

Note that in both these cases our object of interest is the solution space of an algebraic function.

\subsection{General definitions of causal extensions of ACTs}

Formally the construction illustrated above extends a probability model so that it applies to a class of control spaces. First suppose that the tree is saturated, i.e. the only constraints on its primitives are the sum to one conditions. There are two quite different formulations of the causal map. The first expresses the effect of a manipulation on the set of independent random variables $\{X(v): v \in S(\mathcal{T})\}$ used to attach probabilities to the tree. After the manipulation $\left\{V^{*}, V^{* *}\right\}$, for each $v^{*} \in V^{*}$ we replace $X\left(v^{*}\right)$ by a random variable $X^{*}\left(v^{*}, v^{* *}\right)$ such that $\operatorname{Prob}\left(X^{*}\left(v^{*}, v^{* *}\right)=v^{* *}\right)=1$.

There is a more algebraic way of formulating this transformation. For each $v^{*} \in V^{*}$ we perform a sequence of projections of the simplex of probabilities $\Pi\left(v^{*}\right)=\left\{\pi\left(v^{*}, v\right): v \in \mathbb{X}\left(v^{*}\right)\right\}$ on to the face $\left(v^{*}, v^{* *}\right)$. It is easily checked that these projections commute for the saturated model.

Thus in either formulation we note that the manipulation $\left\{V^{*}, V^{* *}\right\}$ maps the primitives $\Pi(\mathcal{T})$ onto $\Pi^{*}(\mathcal{T})$. In symbols we can write that for

$$
\Pi(\mathcal{T})=\cup_{v \in S(\mathcal{T})} \Pi(v) \text { and } \Pi^{*}(\mathcal{T})=\cup_{v \in S(\mathcal{T})} \Pi^{*}(v)
$$

we have a map 


$$
\Pi\left(v^{*}\right) \rightarrow \Pi^{*}\left(v^{*}\right)
$$

for each $v^{*} \in V^{*}$, where $\pi^{*}\left(v^{*}, v\right) \in \Pi^{*}\left(v^{*}\right)$ satisfies

$$
\pi^{*}\left(v^{*}, v\right)= \begin{cases}1 & \text { if } v=v^{* *} \\ 0 & \text { if } v \neq v^{* *}\end{cases}
$$

and whenever $v^{*} \in V$

$$
\Pi\left(v^{*}\right)=\Pi^{*}\left(v^{*}\right)
$$

For a non-saturated model we first construct the causal function of interest $\xi(\Pi)$, using the map above. We then impose all the constraint equations of the non-manipulated ACT. We call this extension of the ACT causally valid for $\xi(\Pi)$ if the context allows that $\xi(\Pi)$ can be legitimately considered as the expectation of a real causal manipulation modelled by $\left\{V^{*}, V^{* *}\right\}$. Note that whether or not $\xi(\Pi)$ is causally valid for $\left\{V^{*}, V^{* *}\right\}$ depends both on the nature of the actual manipulation and whether that manipulation is local in the sense that it leaves unaffected unmanipulated parts of the ACT: for more detailed discussion of these important issues see [21]. We also note that this assumption is always made by other authors of causal algebras: e.g. [18].

\section{Causality, conditional parametrisations and Bayesian networks}

\subsection{Causality on Bayesian network}

There has been considerable interest over the last fifteen years in the study of causality as defined in Section 3.2 for models whose unmanipulated distributions and manipulated distributions can be defined on a Bayesian network $\mathcal{G}$ on a set of random variables $\left\{X_{1}, X_{2}, \ldots, X_{n}\right\}$. A common assumption is that that the causal order and the partial order induced by the graph describing the Bayesian network are compatible [19, 24]. This has more recently been extended to the class of chain graphs by [13]. Suppose each $X_{i}$ in a Bayesian network $\mathcal{G}$ takes values in $\mathbb{X}_{i}$ and we assume $\mathbb{X}_{i}=\left\{1, \ldots, l_{i}\right\}=\left[l_{i}\right]$ for $l_{i} \in \mathbb{Z}_{>0}$. It is then easily checked that for this special case Pearl's definition of manipulating a variable $X_{k}$ to take a value $x_{k}^{* *}$ is a special case of the manipulations we discuss above. The set $V^{*}$ of manipulated situations is associated with a configuration $\left(x_{1}, x_{2}, \ldots, x_{k-1}\right)$ associated with the variables $\left(X_{1}, X_{2}, \ldots, X_{k-1}\right)$ preceding $X_{k}$ in the Bayesian network partial order, i.e. all those situations which are a distance of $(k-1)$ edges from the root vertex of the event tree corresponding to the Bayesian network. Each situation $v^{*} \in V^{*}$ is manipulated to the unique situation $v^{* *}$ corresponding to the event $\left(x_{1}, x_{2}, \ldots, x_{k-1}, x_{k}^{* *}\right)$.

Converting to Pearl's notation in [19] suppose we intervene on the system by manipulating the variable $X_{j}$ to take the value $x_{j}^{* *}$. Let $p\left(\mathbf{x} \| x_{j}^{* *}\right)$ denote 
the probability mass function on $X$ after such intervention, for $\mathbf{x} \in \prod_{i=1}^{n} \mathbb{X}_{i}$. A causal Bayesian network (CBN) asserts not only that the factorisation formulae associated with the Bayesian network hold, but also that

$$
p\left(\mathbf{x}|| x_{j}^{* *}\right)= \begin{cases}0 & \text { if } x_{j} \neq x_{j}^{* *} \\ \prod_{i=1, i \neq j}^{n} \pi\left(x_{i} \mid \mathbf{x}^{(i-1)}\right) & \text { if } x_{j}=x_{j}^{* *}\end{cases}
$$

where

$$
p(\mathbf{x})=\prod_{i=1}^{n} \pi\left(x_{i} \mid \mathbf{x}^{(i-1)}\right)
$$

is the factorization associated with the Bayesian network and for $i=1, \ldots, n$ the random variables $X^{(i-1)}$ are the parents for $X_{i}$ and $\mathbf{x}^{(i-1)}$ are the values they take. Here of course the set $\Pi$ of primitives is simply

$$
\Pi=\left\{\pi\left(x_{i} \mid \mathbf{x}^{(i-1)}\right): \mathbf{x} \in \prod_{i=1}^{n} \mathbb{X}_{i}\right\}
$$

On the basis of Formula (1) Pearl proposed methods for checking whether or not, on the basis of the observed marginal probabilities of subsets of the $X_{1}, \ldots, X_{n}$ (these marginals might be called the set of manifest probabilities), another function - the total cause of $x_{j}$ on $x_{k}$ - defined by

$$
\xi_{j, k}(\Pi)=p\left(x_{k}|| x_{j}\right)=\sum_{x_{i} \in \mathbb{X}_{i}, i \neq k}\left[\prod_{i=1, i \neq j}^{n} \pi\left(x_{i} \mid \mathbf{x}^{(i-1)}\right)\right]
$$

is identified.

Within this rather restricted class of models and manipulations, the graphical characteristics of sometimes very large Bayesian networks are used to determine sufficient conditions for determining when a function $\xi_{j, k}(\Pi)$ of the probabilities of a manipulated system could be expressed as a function of certain observed margins, given that the observed margins were feasible. In algebraic terms these methods used the configurations of stages to guide an elimination to an explicit expression for $\xi(\Pi)$ in terms of the values of the observed quantities. Two well known theorems where $\xi(\Pi)$ is a "total cause" are the Backdoor and Frontdoor theorems, see [19].

In the original formulation of a causal Bayesian network the demand is made that all variables are amenable to manipulation to all of their possible levels and that the manipulation formula outlined above holds true for all such manipulations. It has since been discovered that, although there are some problems where such hypotheses are plausible, these assumptions are unnecessarily restrictive. Actions often involve an intervention only on certain variables [12]. For example we might want to study the effect of manipulating a treatment to a new dose level or the effect of issuing iodine tablets to a 
population before a plume of nuclear contamination arrives overhead. It is not usually necessary to hypothesise a preservation of structure over all possible manipulations but only after a specified intervention on a single variable, changing its value from one preassigned value to another of interest. Even in Pearls' illuminating example about the causal link between smoking and lung cancer in [18] it would be quite legitimate to be interested only in the effects on the probability of cancer of preventing a smoker from smoking (cf. average causal effect on the treated in [19]) and not the effect of forcing a non-smoker to smoke.

We have illustrated above that it is straightforward to represent such manipulations and calculate their predicted distributions after manipulation via a composition of simplicial projections. Consequently we can, at least in principle calculate the effect of such a manipulation. Recently theorems analogous to Pearl's Backdoor Theorem have been proved for the more general class of chain event graph models [21] and [29] using a graph derived from the probability tree and its stages to similarly guide a useful elimination. Here we focus on the algebraic techniques to determine such elimination in the more general class of ACT models.

Thus as done in [5, Ch.8] for the more restricted class of Bayesian networks we use techniques from algebraic geometry to address issues such as feasibility and identifiability but with particular reference to the study of causal effects like analogies to total cause. The general problem of when and how one set of polynomial equations can be written as a function of other polynomials has been studied for hundreds of years, often exploiting various types of symmetries. More recently methodologies for constructively identifying solutions to this general problem have been developed through the theory of computational commutative algebra and implemented in various software (e.g. $[4,6])$. Their application to the identification of statistical models, using Factorisation (2), is now well established together with the advantages of an algebraic formulation see e.g. $[1,2,14,20,9]$. We have recently applied these techniques to help identify a cause $[15,22]$ and will illustrate some of our generalisations below.

The direct parametrisation of the joint distribution of a probability model has been well studied especially for Bayesian networks. However we have argued above that it is a parametrisation in terms of conditional primitive probabilities that not only allows us to generalise the class of Bayesian network model into ones that can algebraically represent all probability tree models but also that causal functions are most naturally defined through projections within this conditional parametrisation. In the next section we formally develop how the two representations relate to one another within the familiar class of Bayesian networks. 


\subsection{Conditioning as a projection}

We start by fixing some notation. For $d \in \mathbb{Z}_{\geq 1}$ the $(d-1)$ standard simplex and the $d$ dimensional unit hypercube are

$$
\begin{aligned}
\Delta_{d-1} & =\left\{u \in \mathbb{R}^{d}: u_{i} \geq 0 \text { for } i=1, \ldots, d \text { and } \sum_{i=1}^{d} u_{i}=1\right\} \\
C_{d} & =\left\{u \in \mathbb{R}^{d}: 0 \leq u_{i} \leq 1 \text { for } i=1, \ldots, d\right\}
\end{aligned}
$$

respectively. In a natural Cartesian coordinate system the vertices of $\Delta_{d-1}$ are the points $(1,0, \ldots, 0), \ldots,(0, \ldots, 0,1)$ and those of $C_{d}$ are identified with a full factorial design at levels 0 and 1.

Let $X=\left\{X_{1}, \ldots, X_{n}\right\}$ be a set of discrete random variables. If each $X_{i}$ has $r_{i}$ states then the sample space of $X$ has $r=\prod_{i=1}^{n} r_{i}$ distinct points. Let $[n]=\{1, \ldots, n\}, \mathbb{X}_{i}=\left[r_{i}\right]$ be the sample space of $X_{i}$ and $S_{X}=\prod_{i=1}^{n}\left[r_{i}\right]$ be the sample space of the random vector $\left[X_{1}, \ldots, X_{n}\right]$. For $J \subset[n]$ set $X_{J}=$ $\left\{X_{i}: i \in J\right\}$ and $X_{J^{c}}=\left\{X_{i}: i \in[n] \backslash J\right\}$.

The set of all joint probability distributions on the $n$ random variables is identified with the simplex $\Delta_{r-1}$ simply by listing the probability of each value taken by the random vector

$$
\left(p(x): x=\left(x_{1}, \ldots, x_{n}\right) \in S\right) \in \Delta_{r-1}
$$

where $p(x)=\operatorname{Prob}(X=x)=\operatorname{Prob}\left(X_{1}=x_{1}, \ldots, X_{n}=x_{n}\right)$. Sometimes we use the notation $\Delta_{X}$ for the simplex of all joint probability distributions on $X$.

The notion of independence of random variables corresponds to the requirement that a point in $\Delta_{r-1}$ belongs to the Segre variety [10]. For example for $n=2$ the independence of binary $X_{1}$ and $X_{2}$ is equivalent to the statement that the point $(p(0,0), p(0,1), p(1,0), p(1,1)) \in \Delta_{3}$ lies on the Segre variety $p(0,0) p(1,1)-p(1,0), p(0,1)=0$. This is the well known condition $\operatorname{det} A=0$ where $A$ is the contingency table for $X_{1}$ and $X_{2}$

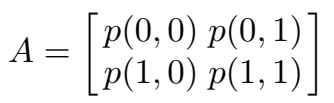

There are various ways to map a simplex into a smaller dimensional simplex. Some are relevant to statistics. Sturmfels (John Van Neumann Lectures 2003) observes that marginalisation over $X_{J}$ and $X_{J^{c}}$ give a linear map which is a projection of convex polytopes. Namely,

$$
\begin{aligned}
m: \Delta_{X} & \longrightarrow \Delta_{X_{J}} \times \Delta_{X_{J^{c}}} \\
(p(x): x \in S) & \longmapsto\left(p_{J}(x): x \in S_{X_{j}}, p_{J^{c}}(x): x \in S_{X_{j^{c}}}\right)
\end{aligned}
$$

where $p_{J}(x)=\sum_{x_{i} \in\left[r_{i}\right], i \in J^{c}} p\left(x_{1}, \ldots, x_{n}\right)$ and analogously for $p_{J^{c}}(x)$. He then shows that the independence model of $X_{J}$ and $X_{J^{c}}$ is the Segre variety on $\Delta_{X}($ see $[8])$. 
Here we use a similar framework to compare the two operations of conditioning and manipulation. The map diagram which we want to study for $n$ binary random variables takes the form in Equation (5)

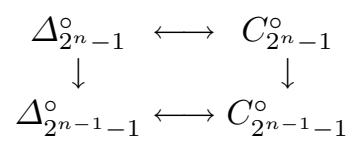

By the multiplication rule the joint probability mass of $X$ factorises according to Equation (6)

$$
\begin{aligned}
& \operatorname{Prob}\left(X_{1}=x_{1}, \ldots, X_{n}=x_{n}\right)= \\
& \operatorname{Prob}\left(X_{1}=x_{1}\right) \operatorname{Prob}\left(X_{2}=x_{2} \mid X_{1}=x_{1}\right) \cdots \\
& \quad \cdots \operatorname{Prob}\left(X_{n}=x_{n} \mid X_{1}=x_{1}, \ldots, X_{n-1}=x_{n-1}\right)
\end{aligned}
$$

given we condition on non-zero probability sets, that is we are outside the boundaries of the simplices involved. For a set $A \subset \mathbb{R}^{d}, A^{\circ}$ is the interior of $A$ with respect to the Euclidean topology. Equation (6) assumes an ordering on the random variables for example we can chose $X_{1}<\ldots<X_{n}$. To simplify notation we rename the parameters on the right hand side of Equation (6) as

$$
\begin{aligned}
\pi_{1}\left(x_{1}\right) & =\operatorname{Prob}\left(X_{1}=x_{1}\right) \\
\pi_{2}\left(x_{2} \mid x_{1}\right) & =\operatorname{Prob}\left(X_{2}=x_{2} \mid X_{1}=x_{1}\right) \\
\vdots & \\
\pi_{n}\left(x_{n} \mid x_{1}, \ldots, x_{n-1}\right) & =\operatorname{Prob}\left(X_{n}=x_{n} \mid X_{1}=x_{1}, \ldots, X_{n-1}=x_{n-1}\right)
\end{aligned}
$$

and Equation (6) becomes

$$
p\left(x_{1}, \ldots, x_{n}\right)=\pi_{1}\left(x_{1}\right) \ldots \pi_{n}\left(x_{n} \mid x_{1}, \ldots, x_{n-1}\right)
$$

Note that

$$
\begin{aligned}
\left(\pi_{1}\left(x_{1}\right): x_{1} \in S_{X_{1}}\right) & \in \Delta_{r_{1}-1} \\
\left(\pi_{2}\left(x_{2} \mid x_{1}\right): x_{2} \in X_{X_{1}}, x_{1} \in X_{X_{2}}\right) & \in \Delta_{r_{2}-1}^{r_{2}} \\
\vdots & \\
\left(\pi_{n}\left(x_{n} \mid x_{1}, \ldots, x_{n-1}\right):\left(x_{1}, \ldots, x_{n}\right) \in S_{X}\right) & \in \Delta_{r_{n}-1}^{\prod_{i=1}^{n-1} r_{i}}
\end{aligned}
$$

The multiplication rule is a polynomial mapping

$$
\mu: \Delta_{r_{1}-1} \times \Delta_{r_{2}-1}^{r_{2}} \ldots \Delta_{r_{n}-1}^{\prod_{i=1}^{n-1} r_{i}} \longrightarrow \Delta_{\prod_{i=1}^{n} r_{i}-1}
$$

defined by

$\left(\pi_{1}\left(x_{1}\right): x_{1} \in S_{X_{1}}, \ldots, \pi_{n}\left(x_{n} \mid x_{1}, \ldots, x_{n}\right): x \in S_{X}\right) \longmapsto\left(p\left(x_{1}, \ldots, x_{n}\right): x \in S_{X}\right)$

for $x=\left(x_{1}, \ldots, x_{n}\right)$ and whose structure is made clearer by an example. For two binary random variables with levels 0 and 1 let 


$$
\begin{aligned}
& s_{1}=\operatorname{Prob}\left(X_{1}=0\right) \\
& s_{2}=\operatorname{Prob}\left(X_{2}=0 \mid X_{1}=0\right) \\
& s_{3}=\operatorname{Prob}\left(X_{2}=0 \mid X_{1}=1\right)
\end{aligned}
$$

then $\Delta_{1} \times \Delta_{1}^{2}$ is isomorphic to $C_{3}$ and

$$
\mu: \begin{aligned}
C_{3} & \longrightarrow \Delta_{3} \\
\left(s_{1}, s_{2}, s_{3}\right) & \longmapsto\left(s_{1} s_{2}, s_{1}\left(1-s_{2}\right),\left(1-s_{1}\right) s_{3},\left(1-s_{1}\right)\left(1-s_{3}\right)\right)
\end{aligned}
$$

The coordinates of the image vector are ordered according to a typical order in experimental design given by taking points from top to bottom when listed analogously to those in Table 3 for $n=3$ and for binary random variables

\begin{tabular}{ccc}
$x_{1}$ & $x_{2}$ & $x_{3}$ \\
\hline 0 & 0 & 0 \\
0 & 0 & 1 \\
0 & 1 & 0 \\
0 & 1 & 1 \\
1 & 0 & 0 \\
1 & 0 & 1 \\
1 & 1 & 0 \\
1 & 1 & 1
\end{tabular}

Table 3. Top to bottom listings of sample points

We note that this map is not invertible on the boundary but it is invertible - through the familiar equations for conditional probability — within the interior of the simplex where division can be defined. For problems associated with the single unmanipulated system this is not critical since such boundary events will occur only with probability zero. However when manipulations are considered it is quite legitimate to ponder what might happen if we force the system so that events that would not happen in the unmanipulated system were made to happen in the manipulated system. It follows that from the causal modelling point of view the conditional parametrisation is much more desirable.

Next consider an $i \in[n]$ and $x_{i}^{* *} \in\left[r_{i}\right]$ such that $\operatorname{Prob}\left(X_{i}=x_{i}^{* *}\right) \neq 0$. Then the conditional probability on $\left\{X_{1}, \ldots, X_{n}\right\}$ given $\left\{X_{i}=x_{i}^{* *}\right\}$ is defined as

$\operatorname{Prob}\left(X_{1}=x_{1}, \ldots, X_{n}=x_{n}\right) \mid X_{i}=x_{i}^{* *}= \begin{cases}0 & \text { if } x_{i} \neq x_{i}^{* *} \\ \frac{p\left(x_{1}, \ldots, x_{n}\right)}{\sum_{x_{-i} \in\left[r_{-i}\right]} p\left(x_{1}, \ldots, x_{n}\right)} & \text { if } x_{i}=x_{i}^{* *}\end{cases}$

in a simplified notation and with $x_{-i}=\left(x_{1}, \ldots, x_{i-1}, x_{i+1}, \ldots, x_{n}\right)$, similarly for $\left[r_{-i}\right]$. Outside the set $x_{i} \neq x_{i}^{* *}$, this mapping is an example of the simplicial 
projection on the face $x_{i}=x_{i}^{* *}$. Indeed, any simplex $\Delta$ in the Euclidean space is the join of any two complementary faces. Moreover faces of simplices are simplices. That is if $F$ and $F^{c}$ are complementary faces, then each point $P$ in the simplex and not in $F$ or $F^{c}$ lies on the segment joining some point $P_{F}$ in $F$ and some point $P_{F^{c}}$ in $F^{c}$, and on only one such segment. This allows us to define a projection $\pi_{F}: \Delta \backslash F^{c} \rightarrow F$, by $\pi_{F}(P)=P_{F}$ if $P \notin F$ and $\pi_{F}(P)=P$ if $P \in F$.

Example \%. For $n=2$ and $P=(p(0,0), p(0,1), p(1,0), p(1,1))$ with $p(0,0)+$ $p(0,1) \neq 0, F=\left\{x \in \Delta_{3}: x=\left(x_{1}, x_{2}, 0,0\right)\right\}$ and $F^{c}=\left\{x \in \Delta_{3}: x=\right.$ $\left.\left(0,0, x_{3}, x_{4}\right)\right\}$, we have

$$
\begin{aligned}
P_{F} & =\frac{1}{p(0,0)+p(0,1)}(p(0,0), p(0,1), 0,0) \\
P_{F^{c}} & =\frac{1}{p(1,0)+p(1,1)}(0,0, p(1,0), p(1,1)) \\
P & =(p(0,0)+p(0,1)) P_{F}+(p(1,0)+p(1,1)) P_{F^{c}}
\end{aligned}
$$

and $P(Y \mid X=0)$ is the operation

$$
\begin{aligned}
\Delta_{3}^{\circ} & \longrightarrow \Delta_{1}^{\circ} \\
(p(0,0), p(0,1), p(1,0), p(1,1)) & \longmapsto \frac{1}{p(0,0)+p(0,1)}(p(0,0), p(0,1))
\end{aligned}
$$

It can be extended to the boundary $\Delta_{1}$ giving for example the probabilities mass functions for which $p(0,0)=0$ or 1 .

Thus algebraically the operation of conditioning returns a ratio of polynomial forms of the type $x /(x+y+z)$ where $x, y, z$ stand for joint mass function values.

By repeated projections we can condition on $\operatorname{Prob}\left(X_{J}=x_{J}^{* *}\right)>0$ with $J \subset[n]$. As before consider the indeterminates $t_{x}$ with $x \in S_{X}$ for the domain space and $b_{y}$ with $y \in\left[r_{-J}\right]$ for the codomain space. The joint probability mass $\left(p(x): x \in S_{X}\right)$ corresponds to $I=\operatorname{Ideal}\left(t_{x}-p(x): x \in S_{X}\right)$ of $\mathbb{R}\left[t_{x}: x \in S_{X}\right]$. In general the set of polynomials in the finite set of indeterminates, $a$ and with coefficients in $\mathbb{R}$ is indicated with $\mathbb{R}[a]$. Its projection onto the $F_{J}$ face of the simplex can be computed by elimination as follows. Let $l$ be a dummy indeterminate and consider $I$ as an ideal in $\mathbb{R}\left[t_{x}: x \in S_{X}, b_{y}: y \in\left[r_{-J}\right], l\right]$. Consider $I+J$ where $J$ is the ideal generated by

$$
\begin{aligned}
& l-\sum_{y \in\left[r_{-J}\right]} b_{y} \\
& b_{y} l-p(x) \sum_{y \in\left[r_{-J}\right]} b_{y}
\end{aligned}
$$

where $x$ and $y$ are suitably matched. Then the elimination ideal of $I+J$ of the $l$ and $t_{x}$ variables corresponds to the simplicial projection. In Example 8 the computation of the simplicial projection in a simple case is performed in the freely available system for computing with multivariate polynomials called $\mathrm{CoCoA}$ [4]. 
Example 8 . The point $P=(1 / 3,1 / 3,1 / 3) \in \Delta_{2}$ is projected onto the face $x_{1}+x_{2}=1$ of $\Delta_{2} . I$ is the ideal of the point $P$ expressed in $t[1], t[2], t[3]$ indeterminates, indeed the only zero of the system of equations $t[1]-1 / 3=$ $t[2]-1 / 3=t[3]-1 / 3=0$ is $P$ itself. $J$ describes a plane parallel to the face $x[3]=0$ of the simplex and $J$ is the ideal in Equation (10). Lex and GBasis are the technical commands that allows the elimination of variables mentioned above.

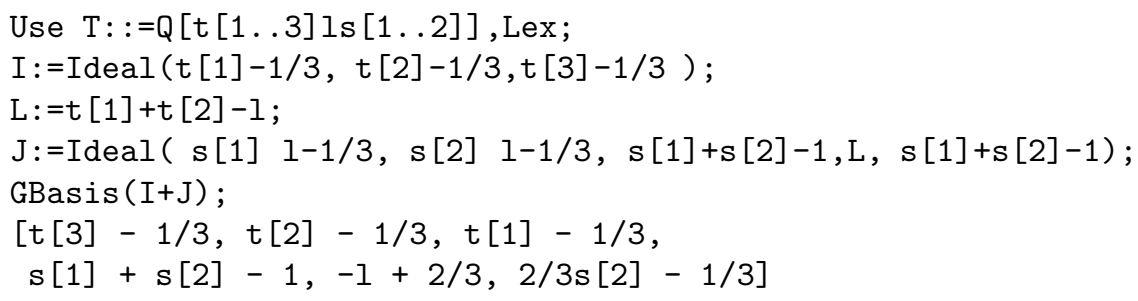

\subsection{The manipulation of a Bayesian network}

In [19] Pearl, starting from a joint probability mass function on $X$, a $x_{i}^{* *}$ and assuming a causal ordering for the intervention $X_{i}=x_{i}^{* *}$, defines a new probability mass function on $X$ given in Equation (2). We have seen above that this operation is best performed in the "conditional" probability parametrisation. In the context of Bayesian networks suppose we let $X_{i}$ denote the variable on which we intervene. Partition $[n]=\{i\} \cup\{1, \ldots, i-1\} \cup\{i+$ $1, \ldots, n\}$ and assume this partition compatible with the causal ordering, that is if $j \in\{1, \ldots, i-1\}$ then $X_{j}$ is not affected by the intervention on $X_{i}$. Take the parametrization

$$
p\left(x_{1}, \ldots, x_{n}\right)=p\left(x_{1}, \ldots, x_{i-1}\right) p\left(x_{i} \mid x_{1}, \ldots, x_{i-1}\right) p\left(x_{i+1}, \ldots, x_{n} \mid x_{1}, \ldots, x_{i}\right)
$$

Then a probability can be seen as a point in

$$
\Delta_{\left[r_{i-1}\right]-1} \times \Delta_{r_{i}-1}^{\prod_{j=1}^{i-1} r_{j}} \times \Delta_{\prod_{j=i+1}^{n} r_{j}-1}^{\prod_{j=1}^{i} r_{j}}
$$

The manipulation operation, restricted to image points for which $x_{i} \neq x_{i}^{* *}$, returns a point in

$$
\Delta_{\left[r_{i-1}\right]-1} \times \Delta_{\prod_{j=i+1}^{n} r_{j}-1}^{\prod_{j=1}^{i-1} r_{j}}
$$

namely the point with coordinates

$$
p\left(x_{1}, \ldots, x_{i-1}\right) \text { and } p\left(x_{i+1}, \ldots, x_{n} \mid x_{1}, \ldots, x_{i}^{* *}\right)
$$

as the $x_{i}$ 's varies. Note that within the conditional parametrisation this is simply a projection: as we saw for the more general class of ACTs. For binary random variables it is the orthogonal projection from $C_{2^{n}-1}$ onto the face $x_{i} \neq x_{i}^{* *}$ which is identified with hypercube $C_{2^{n-1}-1}$. Note that this map is naturally defined over the boundary. In contrast there is no unique map expandable to the boundary of the probability space in $\Delta_{X}$. 


\section{From models to causal ACTs to analysis}

\subsection{The unmanipulated system as a probability graph}

To help compare the class of ACT models with their Bayesian network counterparts we conclude this chapter with an illustration of how certain causal statistical models can be analysed within these two frameworks. We are thus able to demonstrate within a practical framework why a Bayesian network approach is less expressive than its ACT counterpart whilst no easier to study algebraically.

Example 9 (Lighting Circuit). A domestic lighting system has a mains supply that can be tripped off or switched on or off. The mains supply is then cabled to room circuits which in turn can be tripped off or switched on or off. Consider the indicators

$$
\begin{aligned}
& M= \begin{cases}0 & \text { mains supply off } \\
1 & \text { mains supply on }\end{cases} \\
& H= \begin{cases}0 & \text { hall circuit supply off } \\
1 & \text { hall circuit supply on }\end{cases} \\
& K= \begin{cases}0 & \text { kitchen supply off } \\
1 & \text { kitchen supply on }\end{cases} \\
& L= \begin{cases}0 & \text { light } \mathrm{A} \text { in hall fails } \\
1 & \text { light } \mathrm{A} \text { in hall works }\end{cases}
\end{aligned}
$$

There are two logical constraints in this system. Indeed if the mains are off then all power is off and if the hall circuit is off light then A is off. Thus the atomic events of the event space are

$$
\begin{aligned}
& \lambda_{1}=\{M=0\}=\{M=0, H=0, K=0, L=0\} \\
& \lambda_{2}=\{M=1, H=0, K=0\}=\{M=1, H=0, K=0, L=0\} \\
& \lambda_{3}=\{M=1, H=0, K=1\}=\{M=1, H=0, K=1, L=0\} \\
& \lambda_{4}=\{M=1, H=1, K=0, L=0\} \\
& \lambda_{5}=\{M=1, H=1, K=0, L=1\} \\
& \lambda_{6}=\{M=1, H=1, K=1, L=0\} \\
& \lambda_{7}=\{M=1, H=1, K=1, L=1\}
\end{aligned}
$$

The sample space can be represented by the probability tree $\mathcal{T}_{1}$ in Table 9 , where a leaf node has the same label as the corresponding root to leaf path.

Note that the labelling of the edges on the paths corresponds to the sequence defining the path taken in the order $(M, H, K, L)$. Thus for example $\lambda_{6}=\{M=1, H=1, K=1, L=0\}=\left\{\rightarrow^{1} \rightarrow^{1} \rightarrow^{1} \rightarrow^{0}\right\}$.

The situations $V\left(\mathcal{T}_{1}\right)$ of this tree are $S\left(\mathcal{T}_{1}\right)=\left\{v_{i}: 0 \leq 1 \leq 5\right\}$. In this example each situation $v \in S\left(\mathcal{T}_{1}\right)$ labels a random variable $X(v)$ where we assume $X\left(v_{i}\right)$ are independent of each other. Five indeterminates suffice to 


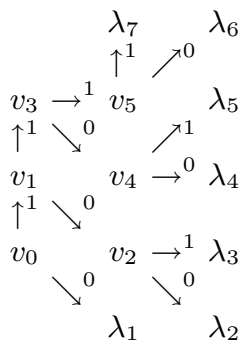

Table 4. A probability tree for the lighting circuit example

describe the primitive probabilities. Indeed write $\pi_{i}=\operatorname{Prob}\left(X\left(v_{i}\right)=1\right), \bar{\pi}_{i}=$ $1-\pi_{i}, 0 \leq i \leq 5$ and $\boldsymbol{\pi}=\left[\pi_{0}, \ldots, \pi_{5}\right]$.

Let $\operatorname{Prob}\left(\lambda_{i}\right)=p_{i}, 1 \leq i \leq 7$ and let $\boldsymbol{p}=\left(p_{1}, p_{2}, \ldots, p_{7}\right)$. Then substituting the sum to one conditions

$$
\boldsymbol{p}=\left[\bar{\pi}_{0}, \pi_{0} \bar{\pi}_{1} \bar{\pi}_{2}, \pi_{0} \bar{\pi}_{1} \pi_{2}, \pi_{0} \pi_{1} \bar{\pi}_{3} \bar{\pi}_{4}, \pi_{0} \pi_{1} \bar{\pi}_{3} \pi_{4}, \pi_{0} \pi_{1} \pi_{3} \bar{\pi}_{5}, \pi_{0} \pi_{1} \pi_{3} \pi_{5}\right]
$$

where we also assume $\left\{1>\pi_{i}>0,1>\bar{\pi}_{i}>0: 0 \leq i \leq 5\right\}$.

One simple way to restrict this model is to impose some conditional independence statements which can always be represented in terms of algebraic constraints. Thus suppose that we believe that:

Item 1. given the mains is on, the supplies in the kitchen and in the hall fail independently, and

Item 2. given the mains and hall lights are on, the kitchen supply and light in hall fail independently.

This implies that $\pi_{5}=\pi_{4}$ and $\pi_{3}=\pi_{2}$ which is easily proved by setting to zero suitable determinants of $2 \times 2$ matrices. Thus we can think of the observed probability vector $\boldsymbol{p}$ as a point in the variety in $C_{7}^{\circ}$

$$
\boldsymbol{p}=\left[\bar{\pi}_{0}, \pi_{0} \bar{\pi}_{1} \bar{\pi}_{2}, \pi_{0} \bar{\pi}_{1} \pi_{2}, \pi_{0} \pi_{1} \bar{\pi}_{2} \bar{\pi}_{4}, \pi_{0} \pi_{1} \bar{\pi}_{2} \pi_{4}, \pi_{0} \pi_{1} \pi_{2} \bar{\pi}_{4}, \pi_{0} \pi_{1} \pi_{2} \pi_{4}\right]
$$

parametrised by the four parameters $\left.\pi_{0}, \pi_{1}, \pi_{2}, \pi_{4} \in\right] 0,1[$.

Computational commutative algebra provides a way to determine the corresponding variety in the $\boldsymbol{\pi}$ variables, that is the set of polynomial relationships that the $\boldsymbol{\pi}$ should satisfy to belong to the conditional model defined in Items 1 . and 2. above. It consists in computing the elimination ideal of the $\boldsymbol{\pi}$ indeterminates for the ideal generated by the seven polynomials associated with the system in Equation (11), namely 


$$
\begin{aligned}
& p_{1}-\left(1-\pi_{0}\right) \\
& p_{2}-\pi_{0}\left(1-\pi_{1}\right)\left(1-\pi_{2}\right) \\
& p_{3}-\pi_{0}\left(1-\pi_{1}\right) \pi_{2} \\
& p_{4}-\pi_{0} \pi_{1}\left(1-\pi_{2}\right)\left(1-\pi_{4}\right) \\
& p_{5}-\pi_{0} \pi_{1}\left(1-\pi_{2}\right) \pi_{4} \\
& p_{6}-\pi_{0} \pi_{1} \pi_{2}\left(1-\pi_{4}\right) \\
& p_{7}-\pi_{0} \pi_{1} \pi_{2} \pi_{4}
\end{aligned}
$$

The actual computation of the elimination ideal is in Appendix 7. The final result and its probabilistic interpretation is given by four polynomials in Table 5 . Note that the third polynomial is simply a consequence of one of our logical constraints.

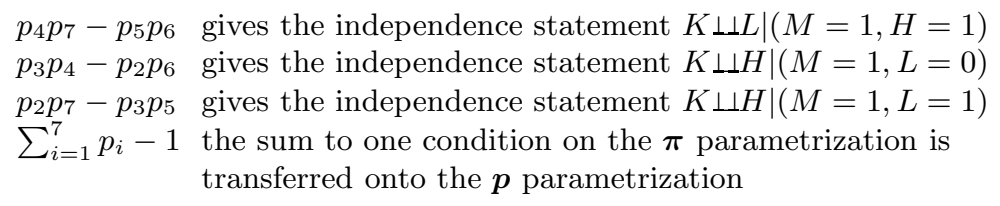

Table 5. The light circuit system expressed in the $\boldsymbol{\pi}$ parameters

\subsection{A Bayesian network for the lighting circuit example}

A more conventional representation of a system with conditional independence constraints is to use a Bayesian network. Here we start with the conditional independence hypotheses on a set of variables defining the process, here $\{M, H, K, L\}$. We might reasonably assert that

1. $H \perp K \mid M$. Thus if the mains is off then we ask that $H$ must be off through our logical constraint whilst if $M=1$ then this is the conditional independence statement in Item 1 . of the previous section.

2. $L \perp\lfloor\mid H, M$. Thus we know by our logical constraint that the lighting circuit is off with certainty (and so independently of $K$ ) unless both the mains and the hall supply are functioning. Furthermore when $M=1=H$ we are in the condition of Item 2. of the last section.

3. $L \sqcup M \mid H$. This is trivially satisfied since $L$ can only be on if the hall supply is on.

Under these assertions, implied by the setting discussed in the last section, we are therefore able to assert that a Bayesian network $\mathcal{G}$ for the lighting circuit is expressed by the graph in Equation (12) below

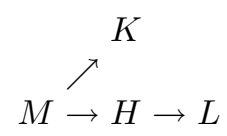


Its implied factorization, for $m, h, k, l \in\{0,1\}$,

$$
\begin{aligned}
& \operatorname{Prob}(M=m, H=h, K=k, L=l)= \\
& \quad \operatorname{Prob}(M=m) \operatorname{Prob}(H=h \mid M=m) \operatorname{Prob}(K=k \mid M=m) \operatorname{Prob}(L=l \mid H=h)
\end{aligned}
$$

is valid. This implies the system can be defined by seven probabilities,

$$
\begin{aligned}
\pi_{1}= & \theta_{M}=\operatorname{Prob}(M=1), \\
\pi_{2}= & \theta_{K \mid M}=\operatorname{Prob}(K=1 \mid M=1), \\
& \theta_{K \mid \bar{M}}=\operatorname{Prob}(K=1 \mid M=0), \\
\pi_{3}= & \theta_{H \mid M}=\operatorname{Prob}(H=1 \mid M=1), \\
& \theta_{H \mid \bar{M}}=\operatorname{Prob}(H=1 \mid M=0), \\
\pi_{4}= & \theta_{L \mid H}=\operatorname{Prob}(L=1 \mid H=1), \\
& \theta_{L \mid \bar{H}}=\operatorname{Prob}(L=1 \mid H=0)
\end{aligned}
$$

with obvious definition of the $\theta$ parametrization. However $\mathcal{G}$ is not a full specification of our problem since our logical constraints force further constraints on the space: namely that

$$
\theta_{K \mid \bar{M}}=\theta_{H \mid \bar{M}}=\theta_{L \mid \bar{H}}=0
$$

This now gives us the identical model space obtained more directly for the ACT. However note that because of the logical constraints the elicitation of this model structure was more involved for the Bayesian network. In particular the known constraints were used not only directly at the end of the elicitation but also earlier when the conditional independence statements were elicited. It is common for causally induced logical constraints to seriously complicate Bayesian networks: so much so that Pearl in his book [19] chooses to preclude them. In contrast the probability tree framework accommodates these constraints automatically. Note that in this example, because of this identification, the algebraic coding of the two models is also identical.

\subsection{Illustrating causal trees and causal Bayesian networks}

Assume that if the system is so insensitive it cannot trip. By this we mean that a light could not cause a circuit to fail, and a failure of the kitchen or hall subsystems could not cause the mains to fail. Call this Model 1. Then we could plausibly argue that the system defined by the ACT above on $\mathcal{T}_{1}$ was causal for the manipulation that forces the hall system to fail by turning it off. Under this causal assertion, under our projection rule above we simply set $\pi_{1}=0$ on the edge $\left(v_{1}, v_{3}\right)$ of $\mathcal{T}_{1}$. The vector of manipulated probabilities $\boldsymbol{p}^{*}$ becomes

$$
p^{*}=\left[\bar{\pi}_{0}, \pi_{0} \bar{\pi}_{2}, \pi_{0} \pi_{2}, 0,0,0,0\right]
$$


Under assumptions like those of Model 1 it might be argued that the Bayesian network $\mathcal{G}$ is also causal, see [19]. This would mean that the projection formula as applied to the Bayesian network would hold for any of its four variables when each is manipulated to either of its two levels. This of course implies that the formula in Equation (13) of the Bayesian network $\mathcal{G}$ will hold in particular for the manipulation turning the hall system off. Under these assumptions we can compute the effect of this intervention by imposing

$$
1=\operatorname{Prob}\left(E_{H}\right)=p_{1}+p_{2}+p_{3}
$$

where $E_{H}=\lambda_{1}+\lambda_{2}+\lambda_{3}$ is the event "the hall circuit is off", and by adjoining the polynomial $p_{1}+p_{2}+p_{3}-1$ to the ideal generator set in Equation (14). The reduced Gröbner basis with respect to the lexicographic term ordering with initial ordering $p_{7}<\ldots<p_{1}<\theta_{L \mid H}<\theta_{K \mid M}<\theta_{H \mid M}<\theta_{M}$ is

$$
\begin{aligned}
& p_{4}, p_{5}, p_{6}, p_{7} \\
& p_{1}+p_{2}+p_{3}-1 \\
& \theta_{M}-p_{2}-p_{3} \\
& \sum_{i=1}^{7} p_{i}-1
\end{aligned}
$$

The first four polynomials correctly set to zero the post intervention probability of events for which $H=0$, that is in the complement event of $E_{H}$. The fifth polynomial is the updated of the sum to one condition written in the given lex ordering. The sixth and last polynomials retrieve the fact that the main and the kitchen circuit are not effected by the intervention on $H$. By the remaining polynomials we deduce that if $\theta_{H \mid M}$ is not zero then $p_{2}=p_{3}=0$, which is possible only if $p_{1}=1$, that is the mains are off.

But note how in this context the causal assumption on the Bayesian network is suspect. In particular what does it mean to manipulate the hall circuit on, when the mains is down. This manipulation is logically precluded by the logical constraints on the model! This illustrates just how restrictive the conditions for a causal Bayesian network can be and demonstrates the need for looser but analogous definitions based on trees.

\subsection{Causal ordering factorisation}

Suppose we are solely interested in extending the probabilistic model in Model 1 so that the corresponding probability tree is causal with respect to the single manipulation of turning the hall circuit off i.e. setting $H=0$. Then there is a probability tree $\mathcal{T}_{2}$ for this problem that is at least as simple as $\mathcal{T}_{1}$ in the sense that all its root to leaf paths are no longer than those in $\mathcal{T}_{1}$ and whose simplicial projection still gives us the required causal polynomials. In the lighting example this tree induces an equivalent but different parametrization of both the original probability space and its causal extension. This tree -introducing variables in the order $(M, K)$ together then $H$ and then $L$ - is given in Table 6 directly associated with the factorisation 


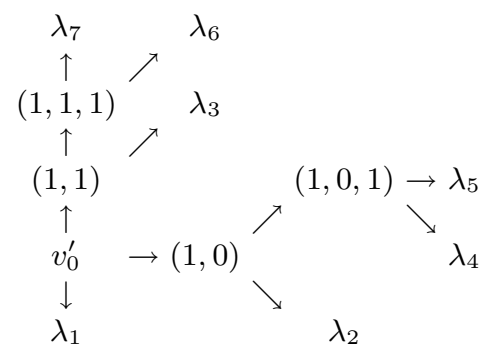

Table 6. An alternative ordering for the light circuit example

$\operatorname{Prob}(M=m, H=h, K=k, L=l)=$

$\operatorname{Prob}(M=m, K=k) \operatorname{Prob}(H=h \mid M=m, K=k) \operatorname{Prob}(L=l \mid M=m, H=h, K=k)$

for $m, h, k, l \in\{0,1\}$. The seven parameters fall into three simplices: parameters upstream of the proposed manipulation, those associated with the manipulation itself (the reference) and the parameters associated with events downstream of the manipulation

Upstream parameters

$$
\begin{aligned}
& \psi_{00}^{u}=\operatorname{Prob}(M=0=K) \\
& \psi_{10}^{u}=\operatorname{Prob}(M=1, K=0) \\
& \psi_{11}^{u}=\operatorname{Prob}(M=1=K)
\end{aligned}
$$

Reference set parameters

$$
\begin{aligned}
& \psi_{0 \mid 10}^{h}=\operatorname{Prob}(H=0 \mid M=1, K=0) \\
& \psi_{0 \mid 11}^{h}=\operatorname{Prob}(H=0 \mid M=1=K)
\end{aligned}
$$

Downstream parameters

$$
\begin{aligned}
& \psi_{0 \mid 110}^{d}=\operatorname{Prob}(L=0 \mid M=1=H, K=0) \\
& \psi_{0 \mid 111}^{d}=\operatorname{Prob}(L=0 \mid M=H=L=1)
\end{aligned}
$$

Thus

$$
\begin{aligned}
& p_{1}=\psi_{00}^{u} \psi_{0 \mid 00}^{h} \\
& p_{2}=\psi_{10}^{u} \psi_{0 \mid 10}^{h} \psi_{0 \mid 100}^{d} \\
& p_{3}=\left(1-\psi_{00}^{u}-\psi_{10}^{u}\right) \psi_{0 \mid 11}^{h} \psi_{0 \mid 101}^{d} \\
& p_{4}=\psi_{10}^{u}\left(1-\psi_{0 \mid 10}^{h}\right) \psi_{0 \mid 110}^{d} \\
& p_{5}=\psi_{10}^{u}\left(1-\psi_{0 \mid 10}^{h}\right)\left(1-\psi_{0 \mid 110}^{d}\right) \\
& p_{6}=\left(1-\psi_{00}^{u}-\psi_{10}^{u}\right)\left(1-\psi_{0 \mid 11}^{h}\right) \psi_{0 \mid 111}^{d} \\
& p_{7}=\left(1-\psi_{00}^{u}-\psi_{10}^{u}\right)\left(1-\psi_{0 \mid 11}^{h}\right)\left(1-\psi_{0 \mid 111}^{d}\right)
\end{aligned}
$$


The $\psi$ indeterminates can be written explicitly as functions of the $\boldsymbol{p}$ by simple application of the definition of conditioning or by computing the Gröbner basis of the ideal corresponding to the polynomial system in Equation (15) with respect to the lexicographic ordering with initial ordering $p_{7}<\ldots<$ $p_{1}<\psi_{7}<\ldots<\psi_{1}$. This computation returns the set of polynomial in Equation (16)

$$
\begin{aligned}
& p_{6}+p_{7}-1+p_{1}+p_{5}+p_{4}+p_{2}+p_{3} \\
& \psi_{0 \mid 111}^{d} p_{6}+\psi_{0 \mid 111}^{d} p_{7}-p_{6} \\
& -p_{4}+\psi_{0 \mid 110}^{d} p_{5}+\psi_{0 \mid 110}^{d} p_{4} \\
& -p_{3}+\psi_{0 \mid 11}^{h} p_{6}+\psi_{0 \mid 11}^{h} p_{7}+h_{1,1} p_{3} \\
& -p_{2}+\psi_{0 \mid 10}^{h} p_{5}+\psi_{0 \mid 10}^{h} p_{4}+\psi_{0 \mid 10}^{h} p_{2} \\
& -p_{5}+\psi_{10}^{u}-p_{4}-p_{2} \\
& \psi_{00}^{u}+p_{2}+p_{3}+p_{4}+p_{5}+p_{6}+p_{7}-1
\end{aligned}
$$

The first polynomial is the sum to one condition, the second and third ones return the downstream parameters, the next two polynomials return the reference parameters and finally the downstream parameters.

Intervention forcing $H=0$ again corresponds to setting conditions $\psi_{0 \mid 10}^{h}=$ 1 and $\psi_{0 \mid 11}^{h}=1$ and thus adjoining the polynomials $\psi_{0 \mid 10}^{h}-1$ and $\psi_{0 \mid 11}^{h}-1$ to the ideal corresponding to the polynomial system in Equation (15). A Gröbner basis of the obtained system is

$$
\begin{aligned}
& p_{4}, p_{5}, p_{6}, p_{7} \\
& p_{1}+p_{2}+p_{3}-1 \\
& \psi_{0 \mid 10}^{h}-1, \psi_{0 \mid 11}^{h}-1 \\
& \psi_{10}^{u}-p_{2} \text { and } \psi_{00}^{u}-1+p_{2}+p_{3}
\end{aligned}
$$

The point here is that the representation of a probability space and its causal extension with respect to a class of manipulations is not usually unique, however they will always agree at least on the interior of their definition space. Which tree is most appropriate will be largely determined by the context. The causal extension of deeper trees, like $\mathcal{T}_{1}$, will permit expression of more causal hypotheses but be less compact algebraically. Thus $\mathcal{T}_{2}$ has paths of length no greater than 3 but is incapable of expressing the manipulation of the kitchen circuit. In contrast, $\mathcal{T}_{1}$ can express this manipulation as a simplicial projection but has paths of length 4 and so is more complex structure. As a general principle we would advocate the encoding of a problem in terms of probability trees with minimal maximum path length sufficient to express all proposed manipulations as a simplicial projection.

\subsection{Trees causation and causal orders}

Much of the early discussion in the literature on graphical expressions of causality was based around the Bayesian network. Since in a Bayesian network any order of how situations happen is subsumed under collections of 
conditional independence statements over variables, causal structures have largely been defined through these cross sectional features. However one of the few transparent properties one might demand about a causal structure is that a cause should happen before an effect. If causal statistical models are based on a single Bayesian network over measurement variables then the contexts to which such models apply are severely restricted: precluding for example causal mechanisms that have the potential to be expressed in both directions. Examples of environments where we would want to express such mechanisms are extremely common. For example in our context we might know that the light failing may trip the hall circuit. So the light failing can cause the hall circuit to trip and the hall circuit failing definitely causes the light to fail.

The solution to this conundrum is simply to focus on describing the system in terms of a probability tree with an associated path event space that is detailed enough to separate different ways in which things can happen as it might pertain to the potential manipulation. Thus the problem with the probability tree and its associated Bayesian network, we have discussed above, is that it implicitly models only the values of the pair of events $(H, L)$, not their timing. This will automatically confound " $\mathrm{H}$ causing $\mathrm{L}$ " with " $\mathrm{L}$ causing $\mathrm{H}$ " if we are in a world where both are possibilities. But it may well be possible to gather information after observing that both failed to help determine whether on observing $(H, L)=(1,1)$ the hall system went first or the light caused the hall system to fail. Thus for example if the light bulb is still intact then it must be off because of a system failure whilst if it has blown then it may well have caused the hall system to trip. The semantics of the tree need to be rich enough to accommodate this type of information when and if it arrives.

It is not hard to draw a new probability tree that expresses not only what happened but in which order situations happened. This gives a richer probability distribution where the atomic events of the original tree form a partition of the atomic events of the new tree. Call this statistical model Model 2. The study of the effects of a given manipulation, as it relates to the behaviour of an unmanipulated system, can then be addressed even for a model like this where causality can be expressed in both directions. Thus for example let $\overline{H K}$ denote the event that "if the hall system fails then the kitchen system fails and the mains doesn't fail". The fact that the light fails is implicit through our logical constraint. Let $\emptyset$ denote no failures. Then, taking account of the logical constraints in the system, the paths of the probability tree giving the corresponding probability tree are

$$
\begin{aligned}
& \rho_{1}=\bar{M}, \quad \rho_{2}=\overline{H M}, \quad \rho_{3}=\overline{H K M}, \quad \rho_{4}=\overline{H K}, \\
& \begin{array}{lll}
\rho_{5}=\bar{H}, & \rho_{6}=\bar{L}, \quad \rho_{7}=\overline{L H M}, & \rho_{8}=\overline{L H K M},
\end{array} \\
& \rho_{9}=\overline{L H K}, \quad \rho_{10}=\overline{L H}, \quad \rho_{11}=\overline{L K M}, \quad \rho_{12}=\overline{L K H M}, \\
& \rho_{13}=\overline{L K H}, \quad \rho_{14}=\overline{L K}, \quad \rho_{15}=\overline{K M}, \rho_{16}=\overline{K H M}, \rho_{17}=\overline{K H}, \\
& \rho_{18}=\overline{K L H M}, \rho_{19}=\overline{K L H}, \rho_{20}=\overline{K L}, \quad \rho_{21}=\bar{K}, \\
& \rho_{22}=\overline{K L M}, \quad \rho_{23}=\emptyset
\end{aligned}
$$


Note that

$$
\begin{aligned}
& \lambda_{1}=\left\{\rho_{1}, \rho_{2}, \rho_{3}, \rho_{7}, \rho_{8}, \rho_{11}, \rho_{12}, \rho_{15}, \rho_{16}, \rho_{18}, \rho_{22}\right\} \\
& \lambda_{2}=\left\{\rho_{4}, \rho_{9}, \rho_{13}, \rho_{17}, \rho_{19}\right\}, \lambda_{3}=\left\{\rho_{5}, \rho_{10}\right\}, \lambda_{4}=\left\{\rho_{14}, \rho_{20}\right\} \\
& \lambda_{5}=\rho_{21}, \lambda_{6}=\rho_{6}, \lambda_{7}=\rho_{23}
\end{aligned}
$$

Write $p=\left(p_{1}, p_{2}, \ldots, p_{24}\right)$ where $p_{i}=\operatorname{Prob}\left(\rho_{i}\right), 1 \leq i \leq 24$. Let for example $\pi_{\bar{H}}^{\bar{L}, \bar{K}}$ denote the probability that the hall system fails given the light and then the kitchen circuit failed. Also write $\bar{\pi}=1-\pi$, and let $\emptyset$ subscript no subsequent failure if such a failure is logically possible. Then the new paths on the probability tree can be given in terms of its edge probabilities as below:

$$
\begin{aligned}
& p_{1}=\pi \bar{M} \\
& p_{4}=\pi_{\bar{H}} \pi \frac{\bar{H}}{K} \pi_{\emptyset}^{\overline{H K}} \\
& p_{7}=\pi_{\bar{L}} \pi \frac{\frac{K}{L}}{H} \pi \frac{\emptyset}{M}
\end{aligned}
$$

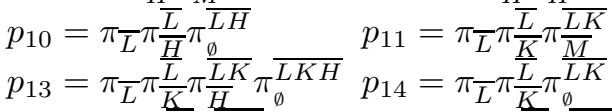

$$
\begin{aligned}
& p_{16}=\pi_{\bar{K}} \pi \frac{\bar{K}}{\bar{H}} \pi \frac{\overline{K H}}{\bar{M}} \quad p_{17}=\pi_{\bar{K}} \pi \frac{\bar{K}}{\bar{H}} \pi_{\emptyset}^{\overline{K H}} \\
& p_{19}=\pi_{\bar{K}} \pi \frac{\bar{K}}{\underline{L}} \pi_{\bar{H}}^{\overline{K L}} \pi_{\emptyset}^{\overline{K L H}} p_{20}=\pi_{\bar{K}} \pi \frac{\bar{K}}{L} \pi_{\emptyset}^{\overline{K L}} \\
& p_{22}=\pi_{\bar{K}} \pi \frac{\bar{K}}{L} \pi \frac{K L}{M} \quad p_{23}=\pi_{\emptyset} \\
& p_{2}=\pi_{\bar{H}} \pi \frac{\bar{H}}{\frac{M}{H}} \quad p_{3}=\pi_{\bar{H}} \pi \frac{\bar{H}}{\bar{K}} \pi \frac{\overline{H K}}{M} \\
& \begin{array}{l}
p_{5}=\pi_{\bar{H}} \pi_{\frac{\emptyset}{H}} p_{6}=\pi_{\bar{L}} \pi_{\frac{\emptyset}{L}}^{\bar{L}} \\
p_{8}=\pi_{\bar{L}} \pi_{\frac{L}{H}} \pi_{\frac{\overline{L H}}{H}} \pi_{\bar{M},}^{\overline{L H K}} p_{9}=\pi_{\bar{L}} \pi_{\frac{L}{H}} \pi_{\emptyset}^{\overline{L H K}}
\end{array} \\
& p_{11}=\pi_{\bar{L}} \pi \frac{\bar{L}}{\frac{K}{L}} \pi \frac{\overline{L K}}{\frac{M}{L K}} \quad p_{12}=\pi_{\bar{L}} \pi \frac{\bar{L}}{\frac{K}{K}} \pi \overline{\frac{L K}{H}} \pi \frac{\overline{L K H}}{M} \\
& p_{15}=\pi \frac{\bar{K}}{\frac{K}{M}} \\
& p_{18}=\pi \bar{K} \pi \frac{\bar{M}}{\bar{L}} \pi \overline{K L} \pi_{\bar{M}}, \overline{K L} \\
& p_{21}=\pi_{\bar{K}} \pi_{\emptyset}^{\bar{K}} \text {, }
\end{aligned}
$$

The semantics of this new tree are much richer and various algebraic identities can be introduced to represent a more specific statistical model and used to embellish this tree as we illustrated with simple examples above. The new tree also helps us to unpick exactly what we mean by manipulating the hall light off. A simple interpretation of this is that we are interested in the manipulation of the hall light off first. This would correspond to manipulating the root vertex, $v_{0}$, of this tree, with emanating edges $(\bar{M}, \bar{H}, \bar{K}, \bar{L}, \emptyset)$ with probabilities $\left(\pi_{\bar{M}}, \pi_{\bar{H}}, \pi_{\bar{K}}, \pi_{\bar{L}}, \pi_{\emptyset}\right)$ to $(0,1,0,0,0)$. This projection allows us to construct the function associated with the total score and examine which functions on this richer tree allow this to be identified. Thus our final point is that the tree and not the Bayesian network is a more natural and flexible framework with which to express the more complicated bidirectional causal structures whilst still coding the problem in a way amenable to algebraic analysis.

\section{Conclusions}

We believe that the natural framework within which causal manipulations of finite discrete models can be studied is the class of ACTs on a probability tree. These encompass a much wider class of models than can be expressed through competing graphical frameworks. They are closed to the type of information 
assumed to be present to embellish a statistical model. Furthermore their parametrisation in terms of conditional probabilities is uniquely suited to this domain, where functions of interest, like the total cause, can be expressed as functions of projections into this space.

Of course many problems still need to be solved. First the nature of the definition of causal functions of the type discussed in this chapter is contentious and their domain of appropriateness not yet fully understood. Second the use of techniques in computer algebra to solve problems of significant size in this domain is a serious challenge because the space of polynomials of interest can be huge. There appears to be a strong need both for more specificity in the class of models considered, and a better understanding of the underlying geometry of these manipulation functions with specific important subclasses of ACTs. However we believe that these issues can be addressed within this model class and that an understanding of the geometry of these algebraic systems could contribute greatly to the study of more general forms of manipulated discrete statistical models.

\subsection{Acknowledgment}

We would like to thank David Mond for many useful discussions that have informed the more technical material in this chapter.

\section{Appendix: maple code}

Here we show how to perform the computation in Subsection 5.1 using the comprehensive computer system for advanced mathematics Maple[6]. Maple, version 6, has two packages to perform Gröbner basis computations, Groebner and grobner with Groebner being the newest. A package is called from the Maple command line with the command with, e.g.

$>$ with(Groebner);

The list of indeterminates for Example 9 is entered below using the Maple command seq

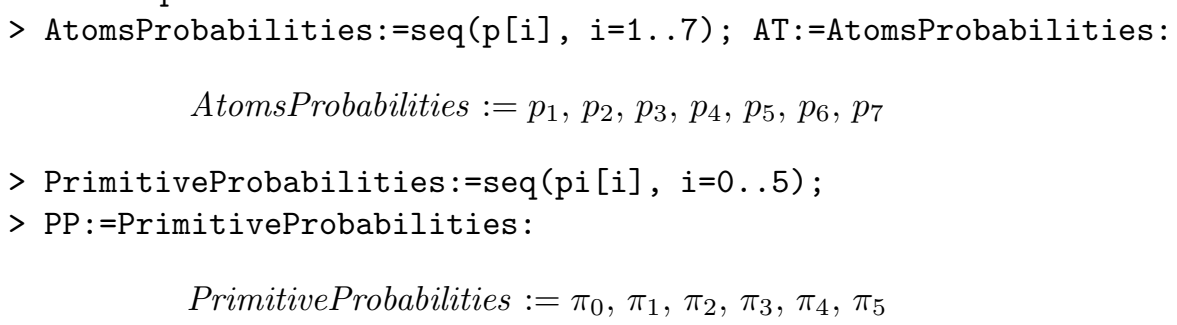

The model we consider is the chain event graph model described in 9 with the two conditions expressed in Items 1. and 2. of Subsection 5.1. To input the polynomial ideal it is sufficient to enter the list of its generators 
$>$ IdealTree:=p[1] $-(1-\mathrm{pi}[0]), \mathrm{p}[2]-\mathrm{pi}[0] *(1-\mathrm{pi}[1]) *(1-\mathrm{pi}[2])$, p [3] -pi [0]*(1-pi [1])*pi [2], p [4]-pi [0]*pi [1]*(1-pi [2])*(1-pi [4]), p [5] -pi [0]*pi [1]*(1-pi [2])*pi [4], p [6]-pi [0]*pi [1]*pi [2]*(1-pi [4]), p [7] -pi [0] *pi [1]*pi [2]*pi [4];

we determine the restrictions imposed on the $p$ by the assumed model with the command

$>$ GB: $=\operatorname{gbasis}(\{$ IdealTree $\}, \operatorname{lexdeg}([\mathrm{PP}],[\mathrm{AP}]))$;

and obtain

$$
\begin{array}{r}
p_{6}+p_{4}+p_{2}+p_{3}+p_{1}-1+p_{5}+p_{7} \\
-p_{7} p_{4}+p_{5} p_{6} \\
-p_{7} p_{2}+p_{3} p_{5} \\
p_{3} p_{4}-p_{2} p_{6} \\
\pi_{4} p_{6}+\pi_{4} p_{7}-p_{7} \\
-p_{5}+\pi_{4} p_{4}+\pi_{4} p_{5} \\
p_{7} \pi_{2}-p_{7}+\pi_{2} p_{5} \\
\pi_{2} p_{4}+\pi_{2} p_{6}-p_{6} \\
\pi_{2} p_{2}+\pi_{2} p_{3}-p_{3} \\
p_{3} \pi_{1}+\pi_{1} p_{6}+\pi_{1} p_{7}-p_{6}-p_{7} \\
\pi_{1} p_{2}+\pi_{1} p_{4}+\pi_{1} p_{5}-p_{4}-p_{5} \\
\pi_{0}-p_{2}-p_{3}-p_{4}-p_{5}-p_{6}-p_{7}
\end{array}
$$

To study the effect of the intervention $H=0$ we adjoin the polynomial $>\mathrm{H}:=\mathrm{p} 1+\mathrm{p} 2+\mathrm{p} 3-1$;

and perform the same type of Gröbner basis computation

$>\mathrm{HGB}:=\operatorname{gbasis}(\{\mathrm{H}, \mathrm{IdealTree}\}, \operatorname{lexdeg}([\mathrm{PP}],[\mathrm{AP}]))$;

obtaining

$$
\begin{array}{r}
p_{7} \\
p_{6} \\
p_{5} \\
p_{4} \\
p_{1}+p_{2}+p_{3}-1 \\
\pi_{0}-p_{2}-p_{3} \\
\pi_{1} p_{3} \\
p_{2} \pi_{1} \\
\pi_{2} p_{2}-p_{3}+\pi_{2} p_{3}
\end{array}
$$




\section{References}

1. Blai Bonet. A calculus for causal relevance. In Uncertainty in artificial intelligence, pages 40-47. Morgan Kaufmann, San Francisco, CA, 2001.

2. Blai Bonet. Instrumentality tests revisited. In Uncertainty in artificial intelligence, pages 48-55. Morgan Kaufmann, San Francisco, CA, 2001.

3. C. Boutilier, N. Friedman, M. Goldszmidt, and D. Koller. Context-specific independence in bayesian networks. In Proceedings of the Twelfth Conference on Uncertainty in Artificial Intelligence, pages 115-123, S. Francisco, CA, 1996. Morgan Kaufmann Publishers.

4. Antonio Capani, Gianfranco Niesi, and Lorenzo Robbiano. CoCoA, a system for doing Computations in Commutative Algebra. Available via anonymous ftp from cocoa.dima.unige.it, 4.0 edition, 2000.

5. e Castillo, J. M. Gutiérrez, and A. S. Hadi. Expert systems and probabilistic network models. Springer, 2002.

6. B. Char, K. Geddes, G. Gonnet, B. Leong, and M. Monogan. MAPLE V Library Reference Manual. Springer-Verlag, New York, 1991.

7. J. J. Forster and P. W. F. Smith. Model-based inference for categorical survey data subject to non-ignorable non-response. Journal of the Royal Statistical Society. Series B. Statistical Methodology, 60(1):57-70, 1998.

8. Luis David Garcia, Michael Stillman, and Bernd Sturmfels. Algebraic geometry of Bayesian networks. J. Symbolic Comput., 39(3-4):331-355, 2005.

9. D. Geiger, C. Meek, and B. Sturmfels. On the toric algebra of graphical models. Annals of Statistics, 34:1463-1492, 2006.

10. Joe Harris. Algebraic Geometry, A First Course. Springer-Verlag, New York, 1992.

11. S. L. Lauritzen. Graphical models. Oxford Science Press, Oxford, first edition, 1996.

12. S. L. Lauritzen. Causal inference from graphical models, chapter in BarndorffNielsen, O.E., Cox, D. R. and Klüppelberg, C. Complex Stochastic Systems, pages 63-107. Chapman and Hall/CRC, London, Boca Raton, 2000.

13. S. L. Lauritzen and T. S. Richardson. Chain graph models and their causal interpretation. Journal of the Royal Statistical Society, Series B, 2002.

14. P. McCullagh. Invariance and factorial models. with discussion and a reply by the author. J. R. Stat. Soc. Ser. B Stat. Methodol., 62(2):209-256, 2000.

15. D. Mond, E. Riccomagno, and J. Q. Smith. Algebraic causality. University of Warwick research report number 393, 2002/3?

16. David Mond, Jim Smith, and Duco van Straten. Stochastic factorizations, sandwiched simplices and the topology of the space of explanations. R. Soc. Lond. Proc. Ser. A Math. Phys. Eng. Sci., 459(2039):2821-2845, 2003.

17. D. Q. Naiman, J. Q. Smith, and H. P. Wynn. Junction tubes and generalised factorization formulae for bayes modelling. University of Warwick research report 395, 2002.

18. J. Pearl. Causal diagrams for empirical research. Biometrika, 82:669-710, 1995.

19. J. Pearl. Causality. Models, reasoning, and inference. Cambridge University Press, Cambridge, 2000.

20. G. Pistone, E. Riccomagno, and H. P. Wynn. Algebraic Statistics, volume 89 of Monographs on Statistics and Applied Probability. Chapman \& Hall/CRC, Boca Raton, 2001. 
21. E. Riccomagno and Jim Q. Smith. The causal manipulation and bayesian estimation of chain event graphs. Technical report, CRiSM Paper (Centre for Research in Statistical Methodology, Warwick), 2005.

22. E. Riccomagno and J.Q. Smith. Non-graphical causality: a generalisation of the concept of a total cause. Technical report, Research report series No. 394, Dept of Statistics, The University of Warwick, 2003.

23. E. Riccomagno and J.Q. Smith. Identifying a cause in models which are not simple bayesian networks. In Proc. Intern. Conf. on "Information Processing and Management of Uncertainty in knowledge-based systems", pages 1345-1322. IPMU, Perugia, 2004.

24. J. M. Robins. Causal inference from complex longitudinal data. In Latent variable modeling and applications to causality (Los Angeles, CA, 1994), pages 69-117. Springer, New York, 1997.

25. James M. Robins, Richard Scheines, Peter Spirtes, and Larry Wasserman. Uniform consistency in causal inference. Biometrika, 90(3):491-515, 2003.

26. R. Settimi and J. Q. Smith. Geometry, moments and conditional independence trees with hidden variables. The Annals of Statistics, 28(4):1179-1205, 2000.

27. G. Shafer. The Art of Causal Conjecture. MIT Press, Cambridge, MA, 1996.

28. B. Theisson, C. Meek, D.M. Chickering, and D. Heckerman. Computarionally efficient methods for selecting among mixtures of graphical models (with discussion). In J. M. Bernardo, J. O. Berger, A. P Dawid, and A.F.M. Smith, editors, Bayesian Statistics 6, pages 631-656, 1999.

29. P. Thwaites and J. Q. Smith. Evaluating causal effects using chain event graphs. In Proceedings of PGM 2006, Czech Republic, September 11-15, 2005.

30. N. Wermuth and S. L. Lauritzen. Graphical and recursive models for contingency tables. Biometrika, 70(3):537-552, 1983. 